XXII.

\title{
Die angeborenen and (nicht operativ) erworbenen Lücken und Spalten des Mesenterium und ihre Bedeutung als Ursachen des Darmverschlusses. \\ Von
}

Dr. W. Prutz,

Privatdocent in Königsberg.

(Mit 2 Abbildungen.)

Nicht ein neuer operativ behandelter Fall gibt, wie sonst bei derlei Zusammenstellungen, Grundlage und damit Rechtfertigung dieser Mitteilung. Aus anderem Anlaß habe ich die Beobachtungen über Lücken und Spalten des Mesenterium zu sammeln gesucht. Was ermittelt, genügt immerhin zu einer Skizze.

Ich schließe dabei aus die operativ entstandenen Defekte, nach Darmresektion und Gastroenterostomie: ätiologisch klar, klinisch teils ohne Besonderheiten gegenüber den anderen Spaltbildungen, teils dem Bilde des Circulus vitiosus näherstehend, schärfen sie nur die eigentlich selbstverständliche Regel ein, durch geeignete Technik sie zu verhüten.

Die angeborenen and spontan oder akzidentell-traumatisch erworbenen Defekte interessieren durch ihre Entstehung und durch die möglichen - keineswegs notwendigen - übeln Folgen, die ihren Trägern erwachsen können.

Abgesehen von der ihrem Zweck entsprechend kurz gehaltenen Darstellung von Wilms kenne ich von über rein kasuistische Betrachtung hinausgehenden Zusammenstellungen nur die von Blecher ${ }^{1}$ ). In entwickelungsgeschichtlicher Beziehung sind sehr

1) Die ältere Zusammenstellung von Besnier (De l'étranglement interne de l'intestin, Paris 1860) war mir nicht zugängllch. Im Dictionnaire encycl. de 
wesentlich die Untersuchungen von Treves, die bisher in der deutschen Literatur, auch der anatomischen, nahezu unbekannt geblieben $\mathrm{zu}$ sein scheinen.

Für einen Teil der hier zu erwähnenden Fälle muß ich die Angaben von Blecher übernehmen, da mir die betreffenden Originale nicht zugänglich waren. Leider ist mir das auch sonst mehrfach begegnet, so daß ein Teil der hier gesammelten $51 \mathrm{Be}-$ obachtungen nur beschränkt verwertbar ist. Das gleiche wird bei anderen Fällen wieder durch die Kürze der Originalmitteilung bedingt.

In der folgenden Übersicht, in der die Auszüge nach Möglichkeit auf das Wesentliche beschränkt sind, sind die Fälle mit Einklemmung eingeteilt in operierte (18) und nichtoperierte (24), der weiteren Einteilung sind die Komplikationen zugrunde gelegt, da sie so am ersten übersichtlich gemacht werden konnten. Angeschlossen sind dann einige (9) Fälle ohne Einklemmung, weil ätiologisch interessant.

\section{A. Operierte Fälle.}

\section{Finklemmung einer freien Schlinge (ohne oder mit Drehung um die Mesenterial- oder Darmachse).}

1. Nélaton (1845). "Zu spät" operiert. Enterostomie: an der vorliegenden Schlinge Zeichen beginnender Peritonitis. „Nach der Operation, die eine vorübergehende Erleichterung gewährte, trat heftige Entzündung auf, der Kranke starb nach 36 Stunden." Sektion: Dünndarm 10 Zoll über dem Cöcum eröffnet. "Als Grund der Einklemmung fand man eine Dünndarmschlinge dicht über dem Cöcum, von einem Riß des Mesenteriums umgeben und eingeklemmt."

2. Ridge und Hilton ${ }^{1}$ ). (Nach Treitz.) 14 jähr. kränklicher Knabe, in den letzten Jahren oft Verstopfung. Erkrankt 6. VIII. 1853 abends plötz-

Méd, 2 ème Sér., T. VII, p. 122 (Paris 1874) erwähnt Besnier, daß er da 12 Fälle von Einklemmungen in "Bauchfellfalten" mitgeteilt habe, davon 8 in Mesenteriallöchern. (Art. ,Mésentère").

1) Treitz (l. c., S. 93 ff.) , bestreitet, daß es sich hier um ein Loch im Mesenterium des Jejunum gehandelt habe, faßt vielmehr den Fall als eine Hernia retroperitonealis auf (vgl. dazu den Fall von de Ha ën, Nr. 22). Ich glaubte ihn hier doch anfnehmen zu sollen, da der Auslegung von Treitz die ganz bestimmten Angaben von Ridge und Hilton gegenüber stehen, die doch nicht weniger Vertrauen verdienen als die anderen. Treitz scheint da seinem Thema zu Liebe in der Auslegung zu weit gegangen zu sein. Außerdem 
lich mit sehr heftigem Schmerz im Leibe, nachdem er vormittags viel gelaufen war. Nachts Erbrechen, Unruhe, Aufschläge, Abführmittel, Einläufe. Am dritten Tage auf letztere reichliche Entleerungen unverdauter Speisereste. Am nächsten Tage wieder Erbrechen. Heimreise. Befund vom Beginn des fünften Tages (Ridge): Elendester Zustand. Bauch unterhalb des Nabels konkav eingesunken. Nur dicht uber und etwas links von dem Nabel sehr empfindliche, gegen das. Epigastrium sich ausbreitende Erhebung, nicht bedeutend, aber von der Einziehung sehr abstechend. Diagnose: vollständige Unwegsamkeit eines Teiles des Jejunum, bedingt durch eine außer dem Darm liegende mechanische Ursache. Erst am nächsten (6.) Tage Operation möglich (Hilton). Medianschuitt. Colon leer, Netz ausgebreitet. Hier und am Dünndarm miliare Tuberkel. Vom linken Rand des Netzes geht ein rabenfederkieldicker Strang mitten durch die kollabierten Dänndarmschlingen gegen die Wirbelsäule an die linke Seite der Wurzel des Gekröses. Durchtrennung mit dem Finger. Er schnürt sicher keinen Darm ein. For. obturatoria frei. Der gegen den Anfang des Jejunum links von der Wirbelsäule aufwärts geführte Finger findet das Hindernis: "Hier zeigte sich, daB da, wo das Jejunum von der Wirbelsäule verhältnismäBig frei wird und mit dem Duodenum zusammenhängt, ein Stück desselben gegen die rechte Seite des Unterleibes hin durch ein abnormes Loch im Mesenterium getreten und daselbst eingeklemmt war. Dieser Teil des Darms wurde nun, durch Ziehen an demselben gegen die linke Seite, aus seiner Lage befreit und nach außen gebracht." Länge etwa 6-8 Zoll $(15-20 \mathrm{~cm})$, nicht gangränös. "In das Loch, durch welches der Darm getreten war, konnte man leicht mit der Fingerspitze eingehen." - Därme unterhalb leer, aber dunkel, mit Blut überfüllt, von ganz eigentlumlichem Aussehen. „Dieses erklärte man sich aus dem Drucke, den die obere Gekrösvene, welche in der Wurzel des Dünndarmgekröses gerade an der Stelle des abnormen Loches sich befand, durch die Spannung und Ausdehnung des Loches erlitten habe..." Tod ca. 8 St. p. op. Keine Sektion.

3. Arbuthnot Lane. 10 jähr. Knabe, früher stets gesund, erkrankt (10. III. 1890) ganz plötzlich im Schlaf mit heftigstem Schmerz im Unterbauch; nach wenigen Minuten Erbrechen. Ol. Ricini, Breiumschlag, Seifenwassereinlauf mehrmals. Am Anfang des dritten Tages Kollaps, Kotbrechen. Bei der Aufnahme moribund. Bauch aufgetrieben, rechter Rektus zeigt unter dem Nabel umschriebene Spannung. Kein Tumor, keine Resistenz. Bauch wenig empfindlich, auch spontan kein Schmerz, vorher dauernd, mit Exazerbationen. Trotzdem Operation: In Narkose deutlicher, großer, vorspringender Tumor am Ort der Muskelspannung. T-Schnitt. Reichlicher, übelriechender, blutiger ErguB. Dunkelblauer Darm, mit Fibrin-

ist er etwas mißtrauisch gegenüber Beobachtungen von Mesenteriallöchern überhaupt. (,Was mich betrifft, so bin ich in Bezug auf diese Löcher, von denen man so vieles zu hören bekommt, sehr ungläubig geworden, nachdem mir unter vielen tausend Leichen, die ich bis jetzt zu untersuchen Gelegenheit hatte, nur zwei Fälle von wirklichen Löchern im Gekröse vorgekommen sind." Das sind die Fälle d) und e) meiner Zusammenstellung. 
niederschlägen bedeckt, liegt vor. Es ist ein etwa $3 \mathrm{FuB}$ (ca. $90 \mathrm{~cm}$ ) langer Dünndarmteil, stark gedehnt, durch ein Loch im Mesenterium „nahe seiner Wurzel" durchgetreten. Dies wird parallel zu den Gefäßen erweitert, der Darm gelöst. Tod während der Operation. - Sektion: Das Loch in ursprünglicher Größe läBt bequem den Zeigefinger durch. Sein Rand ebenso rauh und zottig wie der des Einschnitts, also anscheinend frischen Ursprungs. Das Loch liegt in der Mittellinie, etwa 2 Zoll (ca. $5 \mathrm{~cm}$ ) über der Ileocöcalklappe, und grenzt an die hintere Anheftung des Mesenterium.

Das Loch war offenbar frisch entstanden: vielleicht war ein kleineres schon bestehendes durch den eingetretenen Darm aufgerissen; freilich war der Rand nirgends glatt. (Erklärung von A. L.)

4. Franklin. 37 jähr. Mann erkrankt (8. X. 1893) morgens im Bett plötzlich mit Schmerzen und Erbrechen. Noch eine unbedeutende Darmentleerung, nachher auch auf Klysmen nichts mehr. Schmerzen rechts vom Nabel (wo dann auch das Loch saB). Aufnahme nach $4 \frac{1}{2}$ Tagen, sofort Operation. Über 6 Zoll (ca. 16-17 cm) Dünndarm in einem kreisförmigen Mesenterialloch eingeklemmt, das mit dem Herniotom erweitert wird. Darm portweinrot, mit deutlicher Schnürfurche. Weiter nichts angegeben. Heilung.

5. Greig Smith. 12 jähr. zarter Knabe. Erkrankte etwa 3 Wochen vor der Aufnahme mit plötzlichen Schmerzen und Erbrechen, die ca. 14 Tage anhielten. Danu Besserung, Ende der dritten Woche auf erste feste Nahrung wieder reichliches, grüliches Erbrechen (1. III.). Aufnahme 2 Tage darauf unter der Diagnose ,akuter DarmverschluB“. Bauch stark aufgetrieben, Schlingen gut sichtbar. Erst am folgenden Tag Operation: Das unterste Ileum ist viermal um die Mesenterialachse gedreht. Nach der Rückdrehung findet sich, daß das Darmstück auch noch durch ein Loch im untersten Teil des Dunndarmmesenterium geschlapft und hier eingeklemmt ist. Das vorn quer aber den Stiel der gedrehten Schlinge ziehende Ileum (also Drehung von rechts uber vorn nach links) ist so dünn und blaß, daß es zunächst für den Proc. vermif, gehalten wird, der sich aber in normalem Zustand ganz dicht rechts davon findet. Detorsion. Über SchluB des Loches nichts gesagt. Heilung.

6. Kellett Smith. 15 jähr. Mädchen erkrankt (23. VI. 1897) beim Gehen auf der Straße plötzlich mit Leibschmerzen und Erbrechen. Die Schmerzen lokalisieren sich um den Nabel, sind kontinuierlich, aber mit deutlichen periodischen Steigerungen. Bauch weich und Alach, Bauchatmung. Kleine Dämpfung in der rechten Fossa iliaca, hier auch Druckempfindlichkeit und etwas Resistenz. Operation nach 4 Tagen (27. VI.). K. S. läßt den Darm austreten, bis das Mesenterium gut zu übersehen ist, sucht ihn dann von oben nach unten ab: im unteren Teil des Mesenterium ein Loch von birnförmiger Gestalt. Sein breites Ende entspricht dem Mesenterialansatz einer kleinen Schlinge, die, um die beiden Stellen, wo das Mesenterium wieder normal wird, als feste Punkte sich durch das Loch hindurchgedreht hat. Darm intakt. Naht des Loches. Heilung. - Aussehen der Ränder (smooth thickened margin), Gestalt, Lokalisation des Lochs und Anamnese sprachen sämtlich fär kongenitale Entstehung. 
Die angebor. u. (nicht operat.) erworbenen Lücken u. Spalten d. Mesenter. 403

7. Lutier. 35 jähr. Mann. Vor 6 Jahren Dysenterie und einige Zeit darauf absolute Verstopfung während 21 Tagen. Nie Peritonitis, keine Bauchverletzung. Erkrankt (11. V. 1900) bei Gartenarbeit plötzlich mit starkem Schmerz in der rechten Fossa iliaca. Bald Erbrechen. Kommt nach drei Tagen zu FuB ins Hospital. Verstopfung seit Beginn, aber Entleerung von Flatus. Lokal kein Befund. Tags darauf galliges Erbrechen, Aufstoßen. Am 6. Tag der Krankheit $35 \mathrm{gr}$ Ol. ricini. Abends zwei reichliche Entleerungen. Am nächsten Tag kein Aufstoßen, weiter Erbrechen, dazu jetzt völlige Verhaltung. Schnelle Verschlechterung. Am 8. Tage Operation (in extremis): Medianschnitt. Eiterung in der rechten Fossa iliaca, in der Mitte dieses Herdes Volvulus einer gangränösen Dünndarmschlinge, die zudem noch in einem Mesenterialloch eingeklemmt ist. Völlige Lösung unmöglich, nur Detorsion, Drainage, Anus artific. oberhalb. Tod einige Stunden darauf. - Sektion: allgemeine Peritonitis. Am "Ende“ des Mesenterium, im Winkel zwischen Ileum und Cöcum, das Loch. Sein sichtbarer oberer Rand dick, opak, perlmutterfarben, bildet einen deutlichen Bogen. Nahe dem Cöcum ist dieser sehr viel dicker, durch eine vergrößerte Mesenterialdrasse. Die eingeklemmte Schlinge bedeckt den unteren Rand des Loches. Ihre Schenkel liegen nahe aneinander, zudem ist sie um die Mesenterialachse gedreht. Vom austretenden Schenkel bis zum Cöcum nur zirka $4 \mathrm{~cm}$; dicht über dem eintretenden der Anus artific. Nach Lösung bildet die Schlinge eine lange Schleife, deren Fußpunkte durch das Mesenterium auf zirka $3 \mathrm{~cm}$ genähert sind. Das ist der untere Rand des Loches. Dies ist oval, $6 \mathrm{~cm}$ hoch, $3 \mathrm{~cm}$ breit, die Ränder regelmäßig glatt, scharf, nur an der erwähnten Drüse verdickt. Der obere Rand wird umfaßt von zwei Ästen der Art. mesent. sup.

Die plötzliche Verschlechterung rühre wohl daher, daB (nach dem Abführmittel) sekundär der Volvulus auftrat. - Bezı̈glich der Ätiologie beruft L. sich auf Treves.

8. Blecher. 20 jähr. Mann. Hat fruher öfters an Verstopfung gelitten. Letzter Stuhlgang 13. Dez. Erkrankt 14. Dez. ohne besondere Ursache mit Leibschmerzen, die allmählich zunehmen. In der zweiten Nacht galliges Erbrechen, das anhält. 16. Lazarettaufnahme. Auf Eingießungen einige harte Kotbröckel entleert. Nachts wieder Erbrechen. 17.: Zustand gut, P. 63. Leib über dem Nabel eingesunken, weich. Unterbauch Auftreibung, rechts mehr wie links, nach rechts konvex, nach oben konkav, nach links nicht deutlich begrenzt. Hier vertiefter tymp. Schall. Keine Peristaltik; tuber dem Nabel Gurren. Operation verweigert; EingieBungen. Erbrechen hält au. Auftreibung nimmt zu. 19. Operation. In Narkose nichts Umschriebenes zu fühlen. Medianschnitt. Stark geblähter Dünndarm drängt sich vor; Cöcum und Colon leer. Etwa $5 \mathrm{~cm}$ über seinem Ende ist das Ileum zu einem Strang gedreht, daran eine geblähte Schlinge. „Im Gekröse des untersten Ileums findet sich ein über handtellergroßes Loch; dasselbe wird dadurch gebildet, daß die Mesenterialwurzel nach unten in freien Rand endigt, der arkadenförmig auf einer Strecke von zirka 50 (?) $\mathrm{cm}$ bis zu dieser Schnurstelle zieht, wodurch die entsprechende Ileumschlinge einen teilweise nur $2-3 \mathrm{~cm}$ breiten Mesenterialsaum hat; der Endteil $(5 \mathrm{~cm})$ des Ileums besitzt dann wieder ein eigenes kurzes 
Mesenterium. Durch das Loch hindurch hat nun die dartber liegende Schlinge eine volle Drehung in links gewundener Spirale vollführt." Leichte Rackdrehung; an beiden Enden, bes. am abfuhrenden, deutliche Schnürfurchen. - Rechtsseitige Pneumonie. Heilung.

9. Trendelenburg (v. Ofenheim). 48jähr. Frau. Zuweilen akute Darmstörungen resp. Stuhlverhaltung, zuletzt vor $1 \mathrm{Jahr}$, immer obne Behandlung geschwunden. Vor 17 Tagen (Aufnahme und Operation am 18. Krankheitstage) trat zeitweise Leibschneiden auf, das zunahm, bis etwa am 11. Tage der Stuhlgang ausblieb. Opium? - Schwerer Allgemeinzustand, Temp. 38, Puls 100. Bauch aufgetrieben, deutliche Peristaltik und Kollern. - Gleich Operation: Medianschnitt. Hinter dem nach oben geschlagenen Netz drängen sich stark injizierte Darmschlingen vor, "hinter welchen man eine abnorme Öffnung in Mesenterium fuhlte." "Yach vollständiger Eventration fand man einen schräg von oben nach unten in der Richtung gegen das Cöcum verlaufenden klaffenden Schlitz in dem stark verlüngerten Gekröse des Dünndarmes, weleher von der Radix in der Höhe des Duodenalschenkels bis knapp zum Ansatze des Danndarmes reichte." Mehrere Schlingen des Ileum, das Cöcum und ein Teil des Colon ascendens sind von rechts hinten nach links vorn $\mathrm{zweimal}$ durch den Spalt durchgetreten (also Drehung um zirka 7200). So ist der Darm zu einer Art Strang zusammengedreht und im Vergleich zum zuführenden wenig gebläht. Rückdrehung, worauf der Spalt die beschriebene Form erkennen läbt. Seine Ränder leicht verdickt, aber obne Zeichen von Narbenbildung. Links rom Spalt die oberen vier Fünftel des Dünndarms, rechts von ihm das unterste Fünftel (und Cöcum und Colon ascendens, also Mesent. ileo-coeci comm., wie nach der Beschreibung und bes. nach Taf. II. zweifellos). - Sofort nach der Ruckdrehung geht der Darminhalt nach unten weiter. Naht des Spaltes, „wobei, wie es scheint, die Vena mesenterica superior oder ein größerer Ast derselben angestochell wurde. . . . Nach Auffindung der blatenden Stelle und Unterbindung derselben traten starke Stauungserscheinungen im Mesenterialgebiete des oberen (sic!) Dunndarmes, des Quercolon und Colon descendens auf". Zunächst gunstiger Verlauf, anfangs des 5. Tages p. op. wegen enormen Meteorismus zweite Laparotomie: freies Gas in der Bauchhöhle, Perforation des Cöcum, die zur Anlegung eines Anus artificialis benutzt wird. Tod $1 \frac{1 / 2}{2}$ Tage darauf.

Sektion: Danndarmschlingen stark gebläht, injiziert, nicht getrübt. Colon z. T. mit Gasen und dünbreiigem Kot gefüllt. Fibrinös-eitrige Peritonitis. Schlitz gut geschlossen, keine älteren narbigen Veränderungen an ihm wahrzunehmen. Venen nicht mehr erweitert. Keine Darmgangrän nachweisbar, auch in der Cöcumperforation Schleimhaut nur "gerötet und leicht sukkulent verdickt".

10. Anderson. 14 jähr. Knabe. Erkrankt ohne AnlaB (anBer daB er 2 Tage vorher Rad gefahren ist, sich danach aber über keinerlei Beschädigung beklagt hat) nachts mit Leibschmerzen; $1 / 2$ Stunde darauf Erbrechen, das sich wiederholt, und eine geringe Darmentleerung. $51 / 2 \mathrm{St}$. nach Beginn: blaß, in passiver Ruckenlage, T. 37,2, P. 110; keine Auftreibung, keine Dämpfung, aber Schmerzen bei tiefer Palpation in der 
Die angebor. u. (nicht operat.) erworbenen Lücken u. Spalten d. Mesenter. 405

rechten Fossa iliaca. Kein Kotbrechen. Auf absolute Diät Besserung. 9 Stunden nach Beginn etwas. Meteorismus, bes. in der Blinddarmgegend. Operation verweigert. - Am nächsten Morgen deutliche Auftreibung rechts von der Mittellinie. P. 144. Vorläuf. Diagn.: Appendic. perfor. - Am Abend des zweiten Tages Operation: Schnitt rechts. Sehr reichlicher, stark blutiger ErguB, so daB geradezu an eine Blutung gedacht wird, die vorliegenden Dünndarmschlingen scheinen auch wie mit Blut gefüllt. Proc. vermif. ganz normal. Schnitt nach oben verlängert, wo mehr geblähte Schlingen zu sehen sind. "Beide Bündel" 1) von Schlingen gehen durch ein enges Loch im Mesenterium, das sie stark einschnürt. Lage des Loches im Mesenterium: „an der Basis seiner Anheftung, wo es den II. Lendenwirbel kreuzt." Darm unterhalb normal und leer. Schwierige Einführung einer Leitsonde (director), Erweiterung, Lösung des Darms, Naht des Loches. - Trotz Transfusion Tod wenige Stunden nach der Operation. Das Loch hat feste, abgestumpfte (rounded) Ränder „and appeared to be a natural one". - Sektion: "Sehr geringe Zeichen von Peritonitis". Etwas blutiger ErguB. Darm etwas aufgetrieben, injiziert, mit deutlichen Schnürfurchen. Incarceriert waren etwa $3 \mathrm{FuB}$ (zirka $1 \mathrm{~m}$ ) Ileum. Loch fest durch Naht geschlossen, scheint auch jetzt „to be a natural one".

\section{Einklemmung adhärenter Schlingen.}

11. Kămmell. 60 jähr. relativ kräftiger Mann, „mit den ausgesprochenen Symptomen einer inneren Einklemmung." Zunächst zwei Tage lang Magenspülungen und hohe Eingießungen, subjektive Besserung. Operation: Im Mesenterium ca. $6 \mathrm{~cm}$ langer Schlitz, „durch den die ganze Masse der Därme hindurchgetreten und zum Teil auf der anderen Seite verlötet war. Der Darm war noch gesund, jedoch bestand durch gleichzeitige Achsendrehung und feste Adhäsionen einzelner Partien ein solches Chaos, daB an eine Entwirrung nicht zu denken war." Erweiterung des Schlitzes; Versuch, die Därme herauszuziehen, $\mathrm{mu} B$ wegen Gefahr der Ruptur aufgegeben werden. Wegen Kollaps Durchtrennung des Darms im Hindernis, Einnähung beider Enden. Tod am nächsten Tage. Bei der Sektion zeigt sich die große Schwierigkeit der Lösung der durch das Loch getretenen und zum Teil in ihm fixierten Darmschlingen.

12. Mauclaire. 21 jähr. Mädchen. Seit 5 Tagen kompleter DarmverschluB. Zwischen Nabel und Symphyse eine Resistenz, die an eine Invagination denken läBt. Operation: Einklemmung des Dünndarms in einem Mesenterialloch von der Größe eines Handtellers. Ränder des Risses sehr fest, so daB er für alt gehalten wird. Die eingeklemmte Schlinge durch Adhäsionen an die rechte Fläche des Mesenterium angeheftet, Proc. vermif. an die linke (so daB er vielleicht bei der Einklemmung mitwirkte). Darm in Ausdehnung von $30-40 \mathrm{~cm}$ ganz schwarz. In der Bauchhöhle blutig-seröser Erguß von sehr üblem Geruch. Tod 10 Stunden nach der

1) Die Bezeichnung bezieht sich dem Zusammeuhange nach auf die zanächst vorliegenden und die erst nach Erweiterung des Schnittes sichtbar gewordenen Schlingen. nicht auf Einklemmung von zwei Darmabschnitten. 
Operation. Abbildung des Präparats. - Einige Tage vor Eintritt der Erscheinungen war die Kranke gefallen, was vielleicht von Bedeutung war (d. h. wohl für die Entstehung des Verschlusses).

\section{Einklemmung mehrerer Darmteile.}

13. Lilienthal. (1897.) Mehrere Fuß Dündarm und ein Paket Dickdarm durch ein großes Mesenterialloch (wo?) mit Achsendrehung incarceriert. Bei der Operation besteht schon Peritonitis. Tod am folgenden Tage.

\section{Mit äußeren Hernien komplizierte Fälle.}

14. Billroth. (1866.) 24 jähr. sehr kräftiger Mann erkrankt plötzlich, ohne Gelegenheitsursache, mit heftigen Schmerzen, Erbrechen, Verhaltung. Als Knabe hatte er eine $H$. ing. d. gehabt, die aber lange nicht mehr ausgetreten und auch bei der Aufnahme (am 6. Tage) nicht zu finden war. Diagnose: Ileus, vielleicht doch in Zusammenhang mit der Hernie. Daher Bruchschnitt: Bruchsack leer. Laparotomie nach oben: rote geblähte Schlingen kommen vor. Durch ein abnormes Loch im Mesenterium ist "ein ziemlich langes Stück Darmschlinge" durchgetreten. Beim Versuch der Lösung reißt sie ein. Erweiterung des Mesenteriallochs, Lösung der Schlinge, das Loch in ihr wird zur Enterostomie benutzt. Tod 9 Tage nach der Operation an Bauchwandphlegmone.

15. HinterstoiBer. (Die Krankengeschichte verdanke ich der Güte des Herrn Direktor und Primararzt Dr. H.) 13 jähr. Knabe. Aufg. 22. IV. 1902. Mitteilung des behandelnden Arztes: V. B. kam mittags mit Hernia incarc. dext. int. Besteht seit Samstag, 19. IV., mittags; der Mutter des Knaben soll es aber gelungen sein, selbe Samstag Abend zurück zu bringen. Nachher soll angeblich eine Erleichterung eingetreten sein. Stuhl und Winde gingen jedoch nicht ab. Er hatte zugleich eine inkomplete' Retentio urinae. Wurde katheterisiert. - 22. IV. abends: Bei der Aufnahme bestehen peritonitische Erscheinungen; Bauch trommelartig aufgetrieben, schmerzhaft. Frequenter kleiner Puls (132). Kollabiert. - Da es sich augenscheinlich um Reposition en masse handelt, sofort Operation in Äthernarkose (Dauer der Narkose 2 Std., Verbrauch $140 \mathrm{ccm}$ ). Rechtsseitiger hypogastrischer Schnitt. Bruchsack vorhanden, in demselben Netz. In der Tiefe tastet der Finger einen zweiten einschnürenden Ring. Nachdem die Bruchpforte genügend erweitert worden, kommt brandiger Darm zur Ansicht; der einschnürende Ring erweist sich als rundes, guldenstückgroBes Loch im Mesenterium des Ileum, durch welches der Darm durchgetreten ist und incarceriert wurde. Deutliche Schnürfurchen, peripher knapp am Cöcum. Mißfarbige, übelriechende Flüssigkeit in der Bauchhöhle. Resektion des brandigen Ileum $(40 \mathrm{~cm})$ und des Cöcum. Wegen der bestehenden Peritonitis und des elenden Zustandes des Kranken wird von einer Darmvereinigung abgesehen; die beiden Darmenden werden in die verkleinerte Hautwunde eingenäht. Subkutane Kochsalzinfusion (1 l). - 23. IV. morgens Temp. 37,7, frequenter, nicht zählbarer, kleiner Puls; Benemmenheit. Exitus letalis 22 St. p. op. 


\section{Mit anderwärts lokalisiertem Darmverschluß kompliziert.}

16. Thursfield. 21/2 jähr. Knabe. Auftreibung unterhalb des Nabels, für vergröBerte Mesenterialdrüsen gehalten. Bei der Operation wirl in einem Mesenterialloch eingeklemmter Dünndarm gefunden und das als Ursache des Verschlusses angesehen. Bei der Sektion aber findet sich ein „Volvulus duodeni“, der Dünndarm häıgt an einem dünnen Mesenterialstiel (hanging from a neck of mesentery), d. h. wohl, es besteht ein Mesenterium commune.

\section{Mit Meckelschem Divertikel kompliziert.}

17. A therton. 14jähr. Knabe. Von Geburt an kleine "protrusion" des Nabels. Vom 6. Jahr ab Anfälle von Leibschmerzen, ein- bis zweimal jährlich, einige Stunden bis zwei Tage dauernd, im letzten Jahr mit Erbrechen verlaufend und mit Durchfall endend. Im letzten Halbjahr drei oder vier Anfälle, immer stärker und länger, Ursache vermutlich Diätfehler. Neuer Anfall nach reichlicher Mahlzeit; fünf Stunden nach Beginn: Schlechter Allgemeinzustand, Bauch stark aufgetrieben und tympanitisch, besonders im Epigastrium und um den Nabel. Empfindlichkeit nicht groß, am stärksten um den Nabel, namentlich rechts und unterhalb. Keine Resistenz. - Operation 6 Stunden nach Beginn: Schrägschnitt rechts;

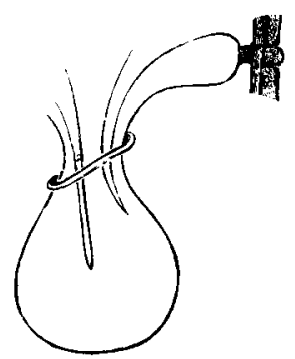

Fig 1 .

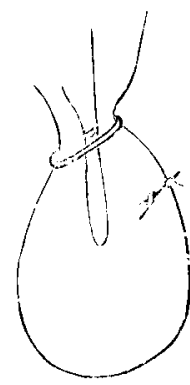

Fig. 2.

Schemata zum Fall von Atherton.

Proc. vermif. gegen die Mittellinie verschoben, hier adhärent. Resektion. Gegen den Nabel hin wirl eine geblähte Darmschlinge gefüblt. Daher Medianschnitt. Ein Meckelsches Divertikel pon Form und Größe einer schlanken Birne mit etwa kleinfingerdickem Stiel, dem Nabel adhärent, an der Basis deutlich gedreht, zum Teil gangränös, viel Kot enthaltend, in diesem ein sehr festes Gebilde von ca. $21_{2} \mathrm{~cm}$ Durchm., wird reseziert. Exzision des Nabels, Bauchnaht. - Tod 34 St. p. op. - Sektion: Eine Ileumschlingo von ca. $1 \mathrm{FuB}$ (etwa $30 \mathrm{~cm}$ ) Länge, ziemlich dunkel gefärbt, aber nicht stark ausgedehnt, tritt durch ein Loch im Mesenterium. Die Schlinge ist mit einigen frischen Verklebungen an den Medianschnitt angelötet. Sie tritt von rechts oben nach links unten durch, ist frei beweglich und leicht zurückzuziehen. Der Divertikelstumpf sitzt an der durchgetretenen Schlinge. Er liegt etwa 6 Zoll $(15-16 \mathrm{~cm})$ über der Ileocöcalklappe. Beide Stümpfe von gutem Aussehen. Darmschlingen in unmittelbarer Nachbarschaft des Mesenteriallochs beträchtlich aufgetrieben. Nirgends ausgesprochene Zeichen von Peritonitis.

Hier ist bei der Operation das Loch gar nicht gefunden worden. A. fügt aber hinzu, es sei ihm aufgefallen, $d a B$ der Darm sich schwer habe vorziehen lassen; daher glaubt er, ihn erst bei der Operation aus dem Loch herausgezogen zu haben. Den Mechanismus stellt er sich folgentermaben 
vor: das Divertikel sei durch die Schlinge, die - je beim Anfall - durchtrat, mitgezogen worden, durch seine Adhäsion am Nabel aber wieder befreit, auch am völligen Durchtreten verhindert worden. Die Verhältnisse lassen sich wohl durch die beiden folgenden Schemata am kürzesten skizzieren.

18. Pye Smith. 14jähriger Knabe, bisher gesund. IBt abends Fische, schläft darnach normal, făhlt sich am nächsten Morgen krank, nimmt keine Nahrung. Um 9 Erbrechen, um 10 Stuhl, mittags nochmals, dann nicht mehr. Leibschmerzen. Weiter Erbrechen in kurzen Pausen während dieses und des năchsten Tages. Bekommt am dritten Tage bei einem Arzneikräuterhändler ein Medikament. Am 4. Tag ärztliche Behandlung: Eingießungen, die etwas Kot entleeren. Die Erscheinungen bleiben gleich. Am selben Abend Aufnahme und Operation: Ein etwas über 2 Zoll $(51 / 2$ bis $6 \mathrm{~cm}$ ) langes Meckelsches Divertikel geht zwischen dem freien und dem Mesenterialrand vom Darm ab, etwa rechtwinklig zu ihm. Dann geht es durch ein Loch in dem Mesenterium des Darmstücks, von dem es entspringt, und umkreist so den Darm dicht oberhalb seines eigenen Ursprunges, indem seine Spitze sich mittels festen Bindegewebes an das Mesenterium ansetzt. So ist der Darm abgeschnürt. - Lösung der Spitze des Divertikels, das dann aus dem Loch herausgezogen wird. Es wird nicht abgetragen, auch über etwaigen Schluß des Loches wird nichts gesagt. - Heilung. - Mit dem ersten Stuhl wird eine Fischflosse entleert. Diese sei zu grob gewesen, den Ring, den das Divertikel bildete, zu passieren, habe quer im Darm gesteckt: auf diese Art sei der Darm in dem einen Durchmesser gedehnt worden, so daß er im anderen zum Schlitz wurde; an den beiden Enden dieses Schlitzes sei das Divertikel abgeknickt worden (weil zu kurz, um die Flosse in Querstellung durchzulassen).' - Zur Diagnose: Der Knabe war zur Invagination zu alt, zum Volv. flex. sigm. und zur Einklemmung eines Gallensteines zu jung, von Fruchtkernen oder einer verschluckten Münze ließ sich nichts ermitteln.

\section{B. Nicht operierte Fälle.}

\section{Einklemmung einer freien Schlinge (ohne oder mit Drehung um die Mesenterial- oder Darmachso).}

\section{J ej un u m.}

19. Adelmann. (1839.) 28jähriger Mann. Vor 4 Jahren ähnliche Erkrankung. Seit 12 Stunden starke Schmerzen und Erbrechen, wegen Meteorismus nichts zu fühlen. Diagnose: wahrscheinlich innere Einklemmung einer ziemlich hohen Darmschlinge. Symptomatische Therapie erfolglos. Tod nach 48 Stunden. Sektion: Kolossaler freier ErguB. „Die mechanische Ursache der Krankheit war eine abnorme Öffnung in der Mesenterialplatte der dritten Windung des Dünndarms, in welcher eine Schlinge (6 Zoll lang) von der überliegenden Windung des Jejunums festgehalten wurde. Ein kleiner Schnitt in den Rand dieser Öffnung löste sie leicht. Daß diese Öffnung eine abnorme war, zeigte das Verhalten des 
Die angebor. u. (nicht operat.) erworbenen Lücken u. Spalten d. Mesenter. 409

Randes, welcher vollkommen glatt ohne Narbe, mit seröser Haut überzogen war." Eingeklemmte Schlinge injiziert, mit Strangulationsmarke.

20. Hirsch. 50jähr. Mann, kräftig, sehr fett. Vor 12 Jahren Sturz vom Wagen, gleich danach heftiger, akuter Schmerz in der Lumbalgegend und im Unterleibe, zugleich heftiges Erbrechen, dann 4 Tage währende Verstopfung. Völlige Erholung erst nach 6 Monaten. Seitdem jährlich zwei- bis dreimal heftige "Erscheinungen einer inneren Incarceration": ohne jegliche nachweisbare Veranlassung Erbrechen, Schmerzen um den Nabel, Verstopfung. Rückgang in 4-5 Tagen auf leichte Abführmittel, am Ende reichliche Darmentleerungen. - Nach sehr kaltem Bad und GenuB kalten Getränks und reichlicher Ausleerung abends leichte Schmerzen, doch Schlaf; am nächsten Morgen Beschwerden stark; Ol. ric. wird erbrochen. Bei der Aufnahme tags darauf schon schlechter Zustand. Bauch überall empfindlich, doch ganz besonders im Mesogastrium 6 cm rechts vom Nabel. Perkussion: Epigastrium und zum Teil auch Mesogastrium tympanitisch, Unterbauch, Colon desc. und Teil des Colon asc, mit mattem Ton. Erbrechen in längeren Zwischenräumen, nicht fälal. Daher Diagnose: "Innere Incarceration wahrscheinlich illı oberen Teile des Dünndarms, vermutlich bedingt durch einen abnormen Strang oder Verlötung von Darmschlingen, als Folge der vor 12 Jahren stattgehabten, traumatischen Peritonitis." Abnorme Öffnung, Riß als sehr selten ausdrücklich ausgeschlossen. - Kalomel und Opium, Einläufe, ohne Wirkung. Tod. Ende des dritten Tages. - In der Mitte des sehr fettreichen Dünndarmmesenterium ein ovales Loch von $12 \mathrm{~cm}$ Umfang, Ränder "etwas callös", nicht dehnbar und vollständig glatt. Von rechts oben ist eine groBe Jejunumschliuge durchgetreten, die dunkle Schnürfurchen zeigt, sich aber leicht herausziehen läßt. Darm oberhalb $1,79 \mathrm{~m}$, eingeklemmtes Stück $1,39 \mathrm{~m}$, Dünndarm darunter $4,49 \mathrm{~m}$. - H. nimmt unter Berufung auf eine Beobachtung von Rokitansky an, daß Fettreichtum des Mesenterium zur Bildung abnormer Öffnungen disponiere.

\section{Ileum.}

21. Brambilla. (Zit. nach Blecher.) „Plötzliche Einklemmung nach heftigem Pressen beim Stuhlgang. Tod nach 3 Tagen. Das rechte Colon herabgesunken und an der vorderen Bauchwand durch ein besonderes Band fixiert. RiB im Mesocolon nahe dem Darm, durch diesen eine ellenlange Ileumschlinge eingeklemmt und gangränös."

22. de Haën. (Nach Treitz, S. 106.) (1766.) 57jähr. Frau, 3 Jahre lang periodische Schmerzen von wechselnder Intensität. Tod unter ähnlichen, nur heftigeren Erscheinungen innerhalb 5 Tagen. Loch in der Mesoflexur, durch das das unterste Ileum durchgetreten und eingeklemmt ist.

Treitz faBt den Fall als Hernia intersigmoidea auf. Er bemängelt die Abbildung von de Haën. Diese kenne ich nur aus der Wiedergabe von Blecher (1. c. S. 57). Danach ist sie freilich nicht sehr übersichtlich. Immerhin scheint mir deutlich: dargestellt ist das untere Ende der Flex. sigm. mit der Umbiegung gegen das Rektum (Colon pelvinum), daran ein Teil der Mesoflexur, und zwar mit der linken Fläche nach dem Be- 
schauer. Also ist das untere Ileum. (dessen über und unter der Einklemmung gelegene Teile ganz deutlich sind), bei nach links gewandter Flex. sigm. von vorn rechts nach hinten links durch das Loch getreten. Letzteres saf im untersten Teil der Mesoflexur, dicht am Fußpunkt des Colonschenkels. Von einer Hernie im eigentlichen Sinn kann nach der Abbildung (eine Beschreibung gibt de Haën nach Treitz nicht) nicht die Rede sein. Außerdem säße eine Hernia intersigm. links von der Flexur. Undeutlich wird das Bild dadurch, da\& der abgebildete Teil der Mesoflexur nach oben gerichtet gezeichnet ist.

23. Heuermann (1778). (Zit. nach Blecher.) „Gestorben an Miserere. Kin Stück des Ileum in eine ringförmige Öffnung in dem Gekröse der Dünndärme eingeklemmt."

24. Palmer. (Sir Astley Cooper, 1. c. S. 210.) (1804.) Frau. Dauer 9 Tage. Massenhafte Abführmittel und Klysmen. Sektion: „Bei genauerer Untersuchung fand sich jedoch eine Öfnnung in dem Mesenterium, welche den unteren Teil des Krummdarmes umschlos, und den traurigen Ausgang des Falles hinreichend erklärte."

25. Albers (1837). (Zit. nach Blecher.) „KinderfaustgroBes Loch im Ileumgekröse nahe dem Cöcum. Durchtritt einer 1\% Fuß langen Schlinge durch das Loch auf die rechte Seite des Mesenterium und Einklemmung. Ileum 3 Zoll oberhalb der Einmündung um die Achse gerreht. Gekröse in der Ungebung des Loches gerötet, Rand sieht frisch gerissen aus."

26. Davies (1844). (Zit. nach Blecher.) „Nach einmal vorübergegangenen Einklemmungserscheinungen 2. Anfall; Tod nach 4 Tagen. 13 Zoll lange Schlinge in ein schillingstückgroßes Loch im Mesocolon eingeklemmt."

27. Spörer (1855). „Den beiden inneren Einklemmungen gingeı mechanische Insulte voraus; der Verlauf der Krankheit war sehr rapit. In dem einen Fall hatte sich eine Schlinge des Ileum von zirka 4 Zoll Länge mit seinem Inhalte durch einen RiB im Mesenterium hindurchgeschoben und eingeklemmt; oberhalb der Einklemmung war der Darm brandig und leicht zerreißlich; die peritoneale Darmentzündung hatte sich auch nach abwärts auf Cöcum und Colon erstreckt."

28. Bauer (1867). 32jähr. Neger. Erkrankung mit heftigen Schmerzen und unaufhörlichem Erbrechen, vorher kopiöser Stuhl, weshall, an Vergiftung gedacht wird. Nicht operiert. „Bei der Sektion wurde ein eingeklemmter Dünndarm in einer Öffnung des Mesenterium, mahe an dessen Befestigung, gefunden."

29. Kaurin (1883). „Der zweite Fall war durch Incarceration einer Dünnlarmschlinge in einer Spalte des Mesenterium verursacht - endete tödlich."

30. Lévèque. (1883). 19 jähr. Manu, bisher gesund, nur seit einem Jahr etwas Verstopfung, erkrankt ganz plötzlich mit heftigsten Kolikschmerzen, besonders um den 'Nabel; arbeitet unter Tags noch; abends Erbrechen, Kommt zwei 'Tage nach Begim zu FuB ins Hospital. Seit 
drei Tagen kein Stuhl: Einläufe. Erbrechen hält an. Befund vom 1uächsten (!) Tag: Puls normal. Bauch mäBig aufgetrieben, intermittierende Schmerzen, durch Druck gesteigert. Palpation negativ. Völlige Verhaltung. Per rectum nichts. Innerlich Eis, potion de Rivière. Eisbeutel, Einläufe. Zunächst etwas besser. Verschlimmerung während der nächsten Woche (!). Zwei bis drei Finger breit über dem Nabel erscheinen zwei etwa olivengroBe platte Körper, vielleicht in der Linea alba eingeklemmtes Netz. Am 13. Tage: enormer Meteorismus, bes. oberhalb des Nabels. Palpation unmöglich. Kotbrechen. Am 15. Tage endlich Freilegung der beiden kleinen Tumoren über dem Nabel: es sind „Fetthernien" (subperitoneale Lipome) in der Linea alba, ohne jeden Zusammenhang mit dem Netz. - Tod: Ende des 18. Tages. - Sektion: Keinerlei ErguB. Nahe dem Nabel entspringt ein fibröser Strang und setzt sich an zwei einander benachbarte und miteinander verwachsene Dünndarmschlingen und das zugehörige Mesenterium an. Hier besteht keinerlei Hindernis, - Ner Dünndarm scheint die ganze Bauchhöhle auszufüllen, ist stark aufgetrieben, stellenweise fein injiziert. Bei Abtrennung vom Duodenum her findet sich beim letzten Fünftel des Dünndarms eine stärker ausgedehnte Partie, unterhalb und etwas rechts vom Nabel gelegen. Hier ist eine Ileumschlinge durch einen „Riß" des Mesenterium getreten. Letzteres ist dicht an seiner Insertion vom Darm abgetrennt, an dem man noch die Ansatzlinie sieht, die an beiden Seiten in das normale Mesenterium übergeht. Länge der Abtrennung $6 \mathrm{~cm}$. Nach hinten verschwindet das Mesenterium in reichlichen Adhäsionen, die es mit den zuerst erwähnten Schlingen verbinden (d. h. den durch den Strang mit dem Nabel verbundenen). Der Darm bildet zwei, das Mesenterium ein Drittel eines Ringes, der das untere Ileumende, dicht vor dem Übergang ins Cöcum, fest einklemmt. Der vom Mesenterium getrennte Darmteil ist um die eigene Achse gedreht, die Drehung ist ganz scharf, liaum $1 \mathrm{~cm}$ lang, am proximalen Ende des Defekts. In dieser Lage ist sie durch feste Adhäsionen fixiert. An der Stelle ist der Darm auBerordentlich verengert, fast wie obliteriert, morsch, reißt ein. Weiter abwärts ist er auf $5 \mathrm{~cm}$ einfach verengert. Dieses Stück, das die vordere Zirkumferenz des Ringes bildet, ist etwa federkieldick; die eingeklemmte Schlinge, die fast einen Kreis bildet, ist $73 \mathrm{~cm}$ lang, $3 \mathrm{~cm}$ unterhalb ihres Austritts aus dem Ring ist der Übergang ins Cöcum. Die Ränder des Risses sind nicht hart und nicht glatt, vielmehr sehr weich und scheinen frisch traumatisch entstanden. Zwischen der eingeklemmten Schlinge und dem Rand des Loches läßt sich die Sonde überall durchführen, außer am oberen und unteren Ende, wo die Schlinge durch Adhäsionen fixiert ist, so daß Detorsion unmöglich. - Ganzer Darm über der Verengerung stark erweitert, die eingeklemmte Schlinge, die übrigens deutliche Schnürfurchen zeigt, hat beinahe normales Kaliber bewahrt.

Nach der wenig übersichtlichen Darstellung ist wohl anzunehmen, daB die durch das Loch getretene Schlinge unmittelbar unterhalb der das Loch tragenden lag; letztere war nicht nur um die Darmachse gedreht, sondern auch selbst stark verengert (was leider nicht näher beschrieben wird). Beides, die Achsendrehung und die Verengerung, könnten den Verschluß bewirkt haben, das Entscheidende war aber wohl die Achsenırehung. 
L.s Besprechung macht die Sache nur noch unklarer: danach sei das völlig Ungewöhnliche an der Beobachtung, daß nur das „eine" Ende „der" Schlinge „eingeklemmt" gewesen sei, während sich das andere ,in den einklemmenden Ring fortsetzt, von dem es einen Teil bildet". Das bezieht sich ersichtlich auf die Schlinge, deren Mesenterium das Loch trug: die wirklich im Loch eingeklemmte war aber $73 \mathrm{~cm}$ lang, jene nur 6, sie war etwas gedehnt, jene stark verengert.

31. Vitrac et Laubie (1897). Leichenpräparat. Tod durch Überfahrenwerden. Zerreißung des rechten Rektus und Psoas, Riß im Mesenterium, in den eine Dünndarmschlinge sich einklemmt.

32. Mitchell (1899). 8 jähr. Knabe, fällt infolge Bruchs des Geländers die letzten Stufen einer Außentreppe herunter, mit dem Leib auf den gefrorenen Boden, besorgt seinen Ausgang aber. Erst zwei Tage darauf erkrankt er mit heftigsten Leibschmerzen. Diagnose: Peritonitis. Nichts gemacht. Tod zwei Tage danach. Sektion (Leichenschau): Loch im Mesenterium nahe dem Übergang' des Ileum in das Cöcum, mit glatten Rändern, darin mehrere Schlingen eingeklemmt. Umschriebene Peritonitis.

33. Parsons (1899). 62 jähr. Mann, an Aorteninsuffizienz leidend, erkrankt, nachdem er 10 Tage lang keinen Stuhlgang gehabt, mit leichtem Übelbefinden im Leibe, nachdem tags vorher etwas galliges Erbrechen gewesen war. Schon mehrere Jahre lang kamen Stuhlverhaltungen von 10-12 Tagen Dauer vor. Bauch nicht aufgetrieben, im Colon Kotmassen zu tasten. Auf Seifenwassereinlauf eine Entleerung, dann nichts mehr. Das Erbrechen wird immer häufiger. Bauch nicht schmerzhaft, weich, keine bemerkenswerte Auftreibung. Schneller Verfall, Tod 6 Tage nach Beginn der Verschlußerscheinungen.

Sektion: Bauchfell hier und da leicht injiziert, einige Exsudatflocken. Darm glänzend (no matting), auch keine alte Peritonitis. Darm nicht besonders ausgedehnt. Im Magen und größeren Teil des Dündarms flüssiger Kot, Colon leer. Im Becken dunkel gefärbte ödematöse Schlingen, die aber ihren Glanz völlig gewahrt haben: die letzten 44 Zoll (zirka $120 \mathrm{~cm}$ ) des Ileum. Sie sind durch ein Loch in einer "anormalen Bauchfellfalte“" durchgetreten, sind aber nicht so fest eingeklemmt, daß sie nicht leicht, ohne Beschädigung, herausgezogen werden könnten.

Die ,abnorme Falte" ist 3 Zoll (zirka $8 \mathrm{~cm}$ ) lang, endet (wie Abb. 2 1. c. zeigt) nach unten mit freiem konkavem Rand. Sie geht von der linken Seite des Beckens an die Flex. sigm., liegt vor dem normalen Mesocolon der Flexur, und zwischen diesen beiden liegt der durch das Loch getretene Darm. Zwischen den „zwei Bauchfellblättern" des abnormen Gebildes (das offenbar deswegen „Falte" genannt wird) laufen Äste der Art. sigmoidea: es handelt sich also um Verdoppelung des Mesosigmoideum. Zwischen ihm und dem regulären Mesosigm. liegt der sechste Teil der Zirkumferenz der Flexur. An seiner Vorderfläche trägt das Gebilde kleine Fettmassen, die sich von App. epiploicae in nichts unterscheiden. AuBerdem hat es zwei ovale glattrandige, je $3 / 4$ Zoll (etwas weniger als $2 \mathrm{~cm}$ ) messende (,congenital") Löcher; durch das untere ist der Darm durchgetreten. - Außerdem: Mäßige Schrumpfniere, Aortenklappen sklerotisch und schluBunfähig. 
Die angebor. u. (nicht operat.) erworbenen Lücken u. Spalten d. Mesenter. 413

Der Tod sei erfolgt an Aorteninsuffizienz, zweifellos beschleunigt durch die Anstrengungen des Erbrechens.

34. Morestin. (Zit. nach Blecher.) "DarmverschluB durch Einklemmung einer Darmschlinge in einer Öffnung des eigenen Mesenteriums." (Soc. anat. 1900 , z. Z. auch mir nicht im Original zugänglich.)

$35^{1}$ ). Im hiesigen pathologischen Institut ist im Jahr 1888 ein Fall von tödlicher Einklemmung in einem Mesenterialloch seziert worden. Die Krankengeschichte fehlt, aus dem Protokoll ergibt sich nur, dab es sich um einen 41 jährigen Mann handelte, daß „in der Fossa subcoecalis" ein längeres Stück Dünndarm durch ein „präformiertes Loch im Mesenterium“, das als „von oben-innen nach unten-außen" verlaufend beschrieben wird, durchgetreten und eingeklemmt sei. (Da die betr. Darmschlingen im kleinen Becken lagen, ist anzunehmen, daB der Darm von rechts oben her durch -das Loch getreten war.) Weiter hat sich über den Fall nichts mehr ermitteln lassen.

\section{Cöcum.}

36. Rokitansky. (Zit. nach Blecher.) Vor drei Jahren plötzlicher Aufall von vorübergehender Einklemmung, seitdem wiederholte Anfälle; 15 Tage nach dem letzten Tod. Im Krummdarmgekröse nächst dem Blinddarm ein drei Zoll im Durchmesser haltendes Loch; durch dieses ist der Blinddarm von hinten nach vorn durchgetreten und nach rechts herumgeworfen, wie ein Kürbis aufgebläht; völlige Abschnürung des Colon an dem Ring.

\section{Colon.}

37. Spörer (1855). „Den beiden inneren Einklemmungen gingen mechanische Insulte voraus; der Verlauf der Krankheit war sehr rapid ... Im zweiten Falle hatte sich ein Teil des Colon descendens mit Inhalt in einem ebensolchen Risse eingeklemmt."

38. Perth. (Zit. nach Lancereaux.) (1878). 35jähr.'Mann, innerhalb 18 Stunden an innerer Incarceration gestorben. Dunndarmschlinge von $7,5 \mathrm{~cm}$ Länge "vom Mesenterium getrennt", letzteres "formant audessous d'elle une corde tendue résistante de $3 \mathrm{~cm} 1 / 2$; dans cette lacune s'était engagée une partie du colon descendant. En un autre point du mésentère existait une seconde lacune semblable, large de $2 \mathrm{~cm} \mathrm{1/2;} \mathrm{mais}$ dans laquelle ne s'était engagé aucun organe."

\section{Einklemmung einer adhärenten Schlinge.}

39. Lancereaux (1872). 40jähr. Frau. Aufgenommen mit Schanker der Oberlippe (in Heilung) und ganz frischer Roseola. Nach drei Wochen abends ganz plötzlich enorme Schmerzen um den Nabel und in den Flanken. Schon nach einer Stunde Bauch aufgetrieben. Zwei Stunden später galliges Erbrechen. Am nächsten Morgen kollabiert, Leib aufgetrieben, bei Perkussion schmerzhaft. Darmschlingen zeichnen sich deutlich durch. Heftige Koliken. Kein Stuhl. Ol. ric., Rhabarber, Wärmflaschen. Tod nach

1) Für die Überlassung des Protokolls bin ich Herrn Geheimrat Prof. E. Neumann zu Dank verpflichtet. 
33 Stunden. Sektion: Dünndarm durch Gas aufgetrieben, in ziemlicher Ausdehnung injiziert; die Hauptsache ist aber die Incarceration eines Teiles des Dünndarms in einem Mesenterialloch. Dies ist für die Faust eines Erwachsenenen durchgängig, länglich, mit glatten, scharfen Rändern, z. T. durch den Rand des zugehörenden Darms gebildet, z. T. durch das Mesenterium. „A l'une de ses angles, l'intestin plié sur lui-même adhère au mésentère épaissi et blanchâtre. Une anse intestinale, d'une longueur de plusieurs décimètres, ecchymosée et distendue par des gaz, se trouvait engagée dans ce trou oì elle s'était étranglée, comme l'indique l'empreinte laissée sur l'intestin au niveau de l'anneau constricteur." Ca. $20 \mathrm{~cm}$ darüber zwei fest verwachsene Schlingen durch etwa linsengroßes Loch in Kommunikation. Weiter hinauf Darm rot, entzündet auf ca. $10 \mathrm{~cm}$.; abwärts ebenfalls injiziert und entzündet, in sehr viel größerer Ausdehnung.

\section{Einklemmuug des größten Teiles des (freien) Dünndarms.}

40. Blandin. (Berichtet von Jobert.) (1823.) „Cet homme, observé à l'hôpital de la Charité, en 1823, par M. Blandin, présentait à son entrée tous les signes d'une violente péritonite, qui se termina rapidement par la mort. A l'autopsie, il rencontre le grand épiploon déjété à gauche, et la presque totalité de l'intestin grèle qui avait franchi l'hiatus de Winslow, énormément dilatée, du reste elle avait ensuite parcouru l'arrière cavité des épiploons, pour en sortir par un trou pratiqué dans le mésocolon transverse."

\section{Einklemmung mehrerer Darmteile.}

41. Strömborg. Schnell verlaufender Fall von Volvulus. "Die Autopsie zeigte Incarceration von SRomanum in eine Öffnung des Mesenterium; auch eine Schlinge vom Ileum war in der Incarceration mit einbegriffen; die Därme waren hyperämisch und von blutigem Exsudat umgeben."

\section{Mit Meckelschem Divertikel kompliziert.}

42. Goltdammer (1889). „Der andere Fall (von innerer Incarccration) - ein Divertikel war durch eine abnorme öfnung des Mesenterium geschlüpft und daselbst eingeklemmt - starb am 4. Tage an Peritonitis" (d. h. nicht operiert).

\section{Fälle ohne Einklemmung. (Nur Mesocolon).}

a) Dittrich (1847). Frau. Perforation eines 4:1-1 $\frac{1 / 2}{2}$ Zoll großen kallösen Ulcus ventriculi in das Duodenum, gefolgt von sekundärer Perforation des Mesocolon transv. „Das Mesocolon transversum war nämlich in seiner Mitte für den Durchgang einer Pomeranze völlig durchbrochen, die Ränder der Durchbruchsstelle kallös, verdickt, wulstig und abgerundet, 
Die angebor. u. (nicht operat.) erworbenen Lücken u. Spalten d. Mesenter. 415

und letztere selbst unmittelbar an der großen Kurvatur des Magens angelagert, und zwar da, wo sich auf der Innenfläche des Magens das Geschwür zeigte und daselbst endigte."

b) Loebl. (Zit. nach Blecher.) Zufälliger Sektionsbefund. Mehr als faustgroßes, elliptisches Loch im Mesocolon transv., begrenzt von den beiden' Art. transv. coli, mit sehnigem Rand, dadurch der gröBte Teil der Dünndärme durchgetreten in die Bursa omentalis.

c) Remboldt. (Zit. nach Blecher.) Bei Sektion eines (?) an Volv. duodeni gestorbenen Kr. „Im Mesocolon links von der Wirbelsäule eine für die halbe Hand durchgängige Spalte, durch welche $2 \frac{1 / 2}{2}$ Fuß Dünndarm frei in die Bursa omentalis getreten sind."

d), e) Treitz fand unter vielen tausend Leichen nur zwei Fälle von wirklichen Löchern im Gekröse. In beiden Fällen saß das Loch in der Mitte des Mesocolon transv. „Durch dasselbe war der größte Teil des Jejunum, jedoch ohne Incarceration, in den Netzbeutel getreten und dadurch das Quercolon tief herabgedrückt. In beiden Fällen war zugleich die hintere Magenwand infolge eines tiefgreifenden chronischen Korrosionsgeschwürs unnittelbar über der Öffnung mit dem Pankreas verwachsen. In diesen Fällen konnte über die Entstehung der abnormen Öffnung kein Zweifel obwalten. - Das Mesocolon transv. ist in die Anwachsung mit hineingezogen, an der angewachsenen Stelle durch den Zug des Quercolon verdünnt und endlich von den andringenden Dünndarmschlingen durchrissen worden." Erwähnt den Fall von Loeb].

\section{Fälle von Defekt im „'Trevesschen Feld" ohne Durchtritt von Darm.}

M.itchell. Unter weit uber 1000 Sektionen (Leichenschau) drei Fälle (auBer dem oben erwähnten):

f) Reife männliche Frucht. Loch im Trevesschen Feld, rund, 1/2 Zoll $(1,27 \mathrm{~cm})$.

g) 24jähriger Mann. Loch auch im „Felde“, elliptisch, 1:3/4 Zoll $(2,54: 1,9 \mathrm{~cm})$.

h) 54jährige Frau. Loch elliptisch, $1: 1 \% / \mathrm{s}$ Zoll $(2,54: 4,76 \mathrm{~cm})$, das "obere" Ende durch eine dünne Membran gedeckt, $3 / 8: 7 / 8$ Zoll $(0,95$ : $2,22 \mathrm{~cm}$ ).

i) Treves. (Sektionsbefund.) 52jühriger Mann. Ovales Loch von $1^{3 / 4}: 1^{1 / 4}$ Zoll $(4,4: 3,2 \mathrm{~cm})$ in seinem „Feld", keine Gefäße, Fett usw. herum, während die Umgebung reichlich Fett zeigt. Rand deutlich opak, verdickt, abgestumpft (abrupt), an der Seite nach dem Cöcum hin von den Endästen der Art. mesent. sup. begleitet. Hier Bauchfell sehr dünn, von ca. 20 Löchern siebartig durchlöchert. „Eine leichte Gewalt konute hier eine ,Incarceration im Mesenterialloch" machen."

Der zweite von Treves beschriebene Fall gehört nicht eigentlich hierher: es handelt sich nicht um einen Defekt des Mesenteriums, sondern um eine GefäBfalte, gebildet von dem letzten Ast der Art. mesent. sup.: 
Reife männliche Frucht. Bei gut ausgebildetem „Feli" hatte der letzte Ast der A. m. s. am cöcalen Rande des Feldes eine Falte gebildet, so daß zwischen dieser und Mlesenterium eine Tasche entstand. die eine förmliche Dohne für eine wandermle Darmschlinge werden konnte, die sie durch diss verdunnte „Feld“* geradezu hindurchleiten konnte.

Das männliche Geschlecht überwiegt bedeutend: von 29 Fällen mit Einklemmung, bei denen das Geschlecht zu ermitteln war, betrafen 23 Männer, nur 6 Frauen; von 5 Fällen ohıe Einklemmung entfallen 3 auf Männer, 2 auf Frauen.

Für die Verteilung anf die Lebensalter sind 26 Fälle von Einklenmung zu verwerten. Im kindlichen Alter (bis zum 14. Jahr) standen 9 M., () W., im mittleren (18 bis 41$) 9 \mathrm{M}$., $3 \mathrm{~W}$., im höheren (48 bis 62) $3 \mathrm{M}$., $2 \mathrm{~W}$.

Von ganz besonderer Bedeutung ist die Lokalisation der Defekte, ihre Verteilung auf die einzelnen Abschnitte des Mesenterium. Sie steht mit der Frage nach der Entstehung der Defekte in innigem Zusammenhang. Dabei sind die Fälle ohne Einklemmung natürlich mitzurechnen.

Auf das Dünndarmmesenterium entfallen 41 Fälle, auf die verschiedenen Abschnitte des Mesocolon 11).

Bei ersterer Gruppe ist leider $17 \mathrm{mal}$ nur allgemein das Dünndarmmesenterium als Sitz des Loches genannt (Fälle von Franklin, Kümmell, Mauclaire, Lilienthal, Billroth, Thursfield, Heuermann, Palmer. Spörer I, Kaurin, Vitrac und Laubie, Morestin. Spörer II. Perth, Lancereaux, Strömborg, Goltdammer).

Alle Fälle mit genaueren Angaben zeigen die ganz auffallende Bevorzugung des untersten Fndes des Dünndarmmesenteriums. Sicher in diesem Bezirk lag das Loch 15 mal (Fälle von Nélaton, Arbuthnot Lane, Greig Smith, Lutier, Blecher, Hinterstoisser, Atherton, Albers, Mitchell, hiesiges patologisches Institut, Rokitansky, dazu ohne Einklemmung: Mitchell [drei Fälle] und Treves). Vermutlich gehören hierher auch die Fälle von Bauer (Loch im Mes. saß „an“ dessen „Befestigung“ d. h. also wohl dicht am Cöcum), Kellett Smith (Loch im ,unteren Teil des Mes.") und vielleicht auch der von Pye Smith (das Loch entsprach dem Teil des Ileum, der das Meckelsche Divertikel trug, das die Finklemmung vermittelt hatte). Sechs Fälle schließlich nehmen hinsichtlich der Lage des Defekts eine Sonderstellung ein: die von Ridge und Hilton (das Loch saß am Anfang des JejunumMesenterium), 'Trendelenburg (ein großer radiärer Spalt teilte 
Die angebor. u. (nicht operat.) erworbenen Lücken u. Spalten d. Mesenter. 417

das Mes. in zwei Abschnitte, von denen der eine zirka is des Dünndarms, der andere das unterste Fünftel des Dünndarms und, als Mes. ileo-coeci comm., Cöcum und Colon ascendens trug), Anderson (der Defekt saß „an der Basis“ des Mes., wo es den II. Lendenwirbel kreuzt), A delmann (im Mes. der dritten Jejunumwindung), Hirsch (in der Mitte des Dünndarmmesenterium), Lévêque (ganz ähnlich wie in dem Fall von Trendelenburg zwischen viertem und letztem Dünndarmfünftel, das Loch war aber von ganz anderer Form, vor allem war der Fall durch eine Verengerung der zum Loch gehörigen Schlinge kompliziert!).

Von den 10 Fällen von Löchern im Mesocolon betreffen nicht weniger als sechs das Mesocolon transversum (Fälle von Blandin, Dittrich, Loebl, Remboldt, zwei von Treitz), einer das Mesocolon ascendens (Brambilla), einer das Mesosigmoideum (de Haën), einmal ist nur Mesocolon ohne nähere Bezeichnung angegegeben (Davies). Ganz ungewöhnlich ist der Fall mit zwei Löchern in einem, „überzähligen" Mesosigmoideum von Parsons.

Ihrer Entstehung nach ist man gewöhnt, die Mesenteriallücken zu scheiden in angeborene und (spontan oder traumatisch) später entstandene. Bei ersteren begnügte man sich sozusagen mit dieser Bezeichnung, ohne sie genauer zu erklären. Trotzdem schien diese Erklärung vor der traumatischen Entstehung den Vorzug zu erhalten: immer wieder begegnet man den $Z$ weifeln gegenüber letzterem Modus. Ich möchte auf Wiedergabe der Literatur hier verzichten und mich an das halten, was aus dem hier vereinigten Material sich ermitteln läßt.

Zunächst haben wir drei kleinere Gruppen von Fällen, die kurz zu erledigen sind: bei zwei ist die Erklärung leicht, bei der lritten scheint es am besten, auf jeden Versuch dazu zu verzichten, da der Erklärung jeglicher reale Boden mangelt. Hierher möchte ich rechnen den Fall von Trendelenburg: radiäre Spaltung des Mesenterium entsprechend der Grenze zwischen viertem und letztem Dünndarmfünftel. Für eine Spalte von solcher Form und solcher Lage wird man aus dem, was wir für die Ontogenese wie die Phylogenese bisher kennen, schwerlich eine Erklärung finden; und mit dem Ausdruck „Entwicklungsstörung“ ist hier im Grunde auch nichts gesagt. Nicht minder verfehlt scheint gegenüber einem solchen Befunde der Versuch. ein Trauma für die Entstehung des Defekts verantwortlich zu machen.

Ähnlich steht es mit dem Fall von Lévêque. Hier lag das Loch genall an der gleichen Stelle des Darms wie im eben er- 
wähnten Fall, war aber, wie schon betont, von ganz anderer Gestalt: 6 cm Darm waren ohne Mesenterium, der mesenteriale Rand des Loches war nur zirka $3 \mathrm{~cm}$ lang (Mes. $1 / 3$, Darm ${ }^{2} ;$ des Umfangs des Loches), das zugehörige Darmstück war verengert. Daß letzteres auf ungenügende Blutversorgung infolge des I)efekts zurückzuführen, ist ja recht walırscheinlich.

Weiter möchte ich hierher zählen die - freilich auch nicht gerade ausführlich berichteten - Fälle von Brambilla und de $\mathrm{Ha} \ddot{\mathrm{e} n}$, sowie den von Adelmann (im Mesenterium der dritten Jejunumwindung.) Wäre über die vielen anderen nur so dürftig beschriebenen Genaueres bekannt, würde diese Gruppe der völlig unklaren Fälle gewiß erheblich größer sein.

Sehr klein ist die Zahl der $z$ weifellos auf traumatische Zerreißung des Mesenterium zurïckzufülrenden Defekte. Mit Bestimmtleit hierher zu rechnen sind nur die Fälle von Hirsch und von Vitrac und Laubie. Hirschs Patient hatte 12 Jahre vor der fatalen letzten Einklemmung ein schweres Bauchtrauma erlitten, dem sehr ernste Erscheinungen und monatelanges Kränkeln folgten; und von da an traten auch die periodisch wiederkehrenden Anfälle von innerer Incarceration auf: hier wird man an dem ursächlichen Zusammenhang schwerlich zweifeln können. Noch klarer liegt der Fall von Vitrac und Laubie: hier war der Riß frisch durch Überfahren entstanden. Allen anderen Fällen wird man iı bezug auf die traumatische Ätiologie höchst mißtrauisch gegenüberstehen müssen. So ist in den Fällen voll Brambilla und Nélaton von einem „Riß" die Rede, in dem Fall von Albers sal - der Rand „frisch gerissen" aus, auch Arbuthnot Lane erwägt. ob der Defekt nicht frisch sei, weil der Rand überall rauh und dick, ganz wie an dem Einschnitt, der zur Lösung des Darms gemacht werden mußte. Arbuthnot Lane wirft die Frage auf, ob nicht vielleicht ein kleineres Loch, das schon vorher bestand (gemeint ist eiu angeborenes wohl im Sinne von Treves), dur(h den eingepreßten Darm weiter aufgerissen sein könnte. Dagegen sprach aber eben, daß sich nirgends am Rande eine glatte Stelle fand. In den beiden Füllen von Spörer schließlich ging (nach dem Referat) "ein mechanischer Insult" der akuten Einklemmung voraus. Der traumatische Insult, der zur Erklärung genügen soll, muß aber ganz besonders Art sein, und ebenso seine Folgen: es kann sich lier nur um schwere Gewalteinwirknngen handeln, und dem müssen auch die Erscheinungen nach der Verletzung entsprechen. Solche Vorgänge müssen in der Anamnese, wie sie der Kranke selbst 
Die angebor. u. (nicht operat.) erworbenen Lücken u. Spalten d. Mesenter. 419

gibt, einen bevorzugten Platz einnehmen, die können nicht gut "vergessen" werden. Es hat geradezu gar keinen Zweck, die Frage, ob traumatisch oder anders entstanden, überhaupt zu erörtern, wenn nicht die Anamnese das schwere Bauchtrauma erweist. Das genügt streng genommen auch noch nicht, denn es könnte ein Loch ja schon vorher dagewesen sein. Also wird weiter auch noch Sitz und Form des Defekts zu berücksichtigen sein. insofern, als bei Sitz des Loches an Stellen, wo sie kongenital öfter vorkommen, wieder zweifelhaft bleiben kann, ob es erst durch das Trauma entstanden. Auch in dieser Beriehung scheint mir der Fall von Hirsch den Anforderungen zu genügen: das Loch traf hier keine der Stellen, an denen kongenitale Defekte, wie gleich zu erörtern sein wird, am ersten zu erwarten sind, vielmehr wäre diese Entstehung angesichts der Lokalisation geradezu unerklärlich. Das Entscheidende ist hier freilich wohl die Anamnese.

Die andere Gruppe von Fällen mit klarer Ätiologie betrifft Defekte des Mesocolon transversum: einwandfrei scheinen hier die Beobachtungen von Dittrich und die beiden von Treitz: es handelt sich hier um Usuren von Magengeschwüren her, die auf die Umgebung übergegriffen haben. Treitz beruft sich auf Loebl, doch habe ich bezüglich dieses letzteren Falles keine Angabe über das Bestehen eines Magengeschwürs tinden können. Ebenso bleibt der Fall von Remboldt nach dem allein verfügbaren kurzen Auszilg unklar.

Vielleicht auch durch eine Art von Usur, freilich nicht durch geschwürige Prozesse, sondern durch das danernde Andrängen eines großen Darmkonvolnts gegen das Mesocolon transversum wird man sich die Beobachtung von Blandin erklären dürfen, wo bei einer Heruie des Foramen Winslowi das ganze Darmpaket durch ein Loch im Mesoc. transv. wieder aus der Bursa omentalis herausgetreten war.

Die Fälle von Dittrich und Treitz stehen außerordentlich nahe dem bisher wohl vereinzelten Fall von Narath'), und zwar scheint da besonders bedentungsvoll, daß es sich hier wie dort immer um Kombination mit alten Magengeschwüren handelt. -

In der großen Mehrzahl der überhaupt genauer berichteten Fälle handelt es sich wahrscheinlich um angeborene Anomalien.

1) Narath, A., Zur Pathologie und Chirurgie der Hernia duodenojejnnalis. Arch. f. klin. Chir., Bd. 71, H. 4. - Einen beinahe gleichen Fall (seinerzeit in der hiesigen medizinischen Klinik beobachtet) kenne ich; er wird an anderer Stelle veröffentlicht werden. 
'Das ergibt sich teils aus der Lokalisation, teils aus dem gehäuften Vorkommen an einer bestimmten Stelle, teils spricht dafür auch die Verbindang der Defektbildung mit anderen Abnormitäten zweifellos kongenitalen Ursprungs.

Zunächst scheint möglich, daß lediglich durch mangellıafte sekundäre Anheftung des Mesenterium an die hintere Bauchwand Lücken zustande kommen. Diese Möglichkeit scheint bisher noch nicht erwogen zu sein.

Dazu müssen wir uns den Verlauf dieser sekundären Anheftung des Mesenteriums kurz vergegenwärtigen. Ich folge dabei der bekannten Darstellung von 'l'oldt.

Augelegt ist das ganze Magendarmgekröse (das spätere große Netz ist das ursprüngliche dorsale Mesogastrium, also eine echte mesenteriale Bildung, ebenso wie das kleine Netz dem beim Menschen nur mehr rudimentär zur Anlage kommenden ventralen Mesenterium entstammt) als eine in allem Anfang median gestellte Platte, die die Anlage des Darmrohrs mit der hinteren Leibeswand verbindet und die ernährenden Gefäße ihr zuführt. Von einem .Einschluß" des Darmrohrs in eine "Duplikatur" des Bauchfells ist keine Rede: erst in viel späterer Zeit, gegen Ende der intrauterinen Entwicklung, wird durch besondere Anordnung des Bindegewebes das Peritonealepithel mit der es tragenden obersten Bindegewebsschicht gegen den die Gefäße usw. bergenden Teil der Gekrösplatte verschieblich, so daß an Stellen, wo die bindegewebigen Elemente in der Umgebung der Gefäße gegen diese ganz zarücktreten, wie z. B. in einem fettfreien Dünndarmmesenterium. allerdings der Eindruck der .,Zweiblätterigkeit" erweckt wird. Die Ortsveränderungen, die es bis zur Erreichung eines der normalen Typen der Darmlage durchzumachen hat, macht das Mesenterium als einheitliche Platte durch. Damit soll keineswegs ausgeschlossen werden, daß bis zur endgültigen gegenseitigen Lagerung der einzelnen Teile zueinander zum Schluß nicht auch ., Verschiebungen" vorkommen, von denen man auch sagen könnte, das eine Orgaı "nähme" einen Teil vom "Bauchfellüberzug" des benachbarten „für sich in Anspruch". Daß derartige Verschiebungen -. wenn auch nicht in solcher Art und solchem Umfange, wie z. $B$. Joh. Müller für das dorsale Mesogastrium annahm, oder wie 'T'reves sie zur Erklärung der endgültigen Lagerung des Darms lieranzieht - vorkommen, ist höchstwahrscheinlich. Sie müssen wohl oder übel herangezogen werden zur Erklärung der Entstehung von Bildungen, die sicher von Anbeginn an keine Be- 
ziehungen zu den primitiven Gekrösen hatten, später aber in Verbindung mit echt mesenterialen Bildungen getroffen werden, Rolle und damit Wert dieser haben und, am fertigen Körper, auch nicht von ihnen zu trennen sind. Für die Frage, ob Mesenteriallöcher auf Mängeln der sekundären Fixation des Gekröses beruhen können, kommt diese Kontroverse nicht in Betracht - obwohl sie vielleicht für eine andere Art der Entstehung von Mesenteriallöchern zu berücksichtigen wäre.

Der am dorsalen Mesenterium in ganzer Länge befestigte Darıl gliedert sich früh in drei scharf geschiedene Abschnitte, die den Gebieten der drei großen Darmarterien entsprechen. Hier kommt nur der zweite Abschnitt in Betracht, die sog. Nabelschleife, die dem späteren Jejunum, Ileum, Colon ascendens und transversum entspricht. Sie tritt zu der Zeit, wo die ersten Anzeichen der beginnenden "Drehung" des Darmes zu bemerken sind - beim etwa sechswöchentlichen Embryo - in steilem Bogen weit aus der Bauchhöhle heraus. Der aufsteigende (rückläufige) Schenkel trägt innerhalb der Nabelscheide, etwa auf der Grenze zwischen seinem äußeren und mittleren Drittel, das als kleine Ausbuchtung angelegte Cöcum. Das Stück vom Beginn der Nabelschleife bis hierher ist es, dessen Ortsveränderungen hier in Frage kommen. Der aufsteigende Schenkel der Nabelschleife kreuzt den absteigenden zunächst, indem er links an ihm vorbei nach oben geht, überkreuzt ihn dann in querer Richtung, so daß der ursprünglich unten befindliche (Colon-)Teil der Schleife von links her über die Basis, den Fußpunkt, des oberen Teils nach rechts tritt: die Kreuzung des Quercolon über das Ende des Duodenum. Hier findet - darin scheinen alle Untersucher übereinzustimmen - eine frühe Fixation statt: der Punkt des Colon, der - durch Vermittelung seines Gekröses - hier sozusagen stationiert wird, verändert in der Folge seinen Ort nicht mehr.

Die Ortsveränderungen, die der Dickdarmteil des nach rechts heriuber getretenen Abschnittes bis zum Anlangen des Cöcum in der rechten Fossa iliaca durchmacht, können hier übergangen werden: wichtig ist nur, daß eben das Mesenterium aller in Betracht kommenden Abschnitte alle Ortsveränderungen mitmacht - welcher der „führende" Teil ist, welche „Kraft" da überhaupt wirkt, ist ganz gleichgültig. Der ursprüngliche Ansatz des Mesenterium an der dorsalen Leibeswand bleibt auch unverändert, nur die relativen Dimensionen der einzelnen Abschnitte erleiden gewisse Änderungen. Der ursprüngliche dorsale Ansatz des gesamte 
Düundarmmesenterium beschränkt sich jetzt anf den unverhältnismäßig schmalen Raum zwischen dem unteren Rand des Pankreas (das ja im dorsalen Mesogastrium entsteht, also einem löheren Gekrüseabschnitt angehört) und dem oberen Rand der Flexura duodeno-jejunalis. Nur dies ist ursprüngliches dorsales Dünndarmmesenterium, alles was kaudalwärts von der Flexura duodenojejunalis liegt, bis zum unteren lleumende - gleichgültig, wieweit nach unten die Fixation reicht - ist nur sekundär an der hinteren Banchwand fixiert. Die Linie, die diese sekundäre Radix mesenterii einhält, zeigt ja verschiedene, z. T. typische Verlaufsarten. die durch weitverbreitete Abbildungen bekannt sind. Mesenteriallücken, die dicht an der hinteren Bauchwand in dem Gebiet der sekundären Radix mesenterii liegen. können anf teilweisem Ausbleiben dieser sekundären Fixation beruhen. So gut letztere manchmal ganz, manchmal zum größeren oder geringeren 'Teil ausbleibt - so entstehen die verschiedenen Grade des Mesenterium conmuue - . ebensogut kann sie auch mal nur auf einem. 'Teil der Linie ausbleiben. Und gerade für diese partiellen Mängel der sekundären Anheftung könnte man sich vielleicht noch eher eine einfache mechanischt Ursache denken, als für die totalen: zu der Zeit, in der sich ein solcher Vorgang abspielen müßte. ist das Längenwachstum dus Darms schon erheblich vorgeschritten, der Dünndarm stark geschlängelt; zu eben der Zeit nimmt die Leber noch einen unverhältnismäßig großen Teil der Bauchloöhle ein, der Darm könnt: also relativ leicht "beengt" werden: eine an die hintere Bauchwand in den Bereich der Fixationslinie ausgewichene Dünndarmschlinge könnte Ursache werden, daß an diesem Punkt die sekındäre Fixation des Mesenterium an der hinteren Bauchwand ausbleibt.

Nächste Voraussetzung für die Berechtigung dieser Vermutung wäre nun, daß Mesenteriallïcken mit entsprecliender Lokalisation vorkommen. Das Loch muß nahe der. hinteren Bauchwand, also im Bereich der sekundären Radix mesenterii, liegen, nicht nahe dem Iarm. Angaben über die Lage, die diese Ientung zu erlauben scheinen, finde ich in folgenden Fällen:

1. Arbuthnot Lane, las Loch liegt in der Vittellinie, 5 ( $\mathrm{m}$ ülocr der Ileocöcalklappe (beim Kinde!), grenzt an die hintere Anheftung des Mesenterium.

2. Blecher, dic Mesenterialwurzel „endet nach unten mit einem freien Rand", rlas bindstück des lleum hat dann wieder ein eigenes kurzes Mesenterium. 
Die angebor. u. (nicht operat.) erworbenen Lücken u. Spalten d. Mesenter. 423

3. Anderson: das Loch lag ,an der Basis der Anheftung“ des Mes., da, wo es den zweiten Lendenwirbel kreuzt.

4. Nicht ausgeschlossen scheint, daB der Fall von Ridge und Hilton hierher gehört: die Lücke lag im obersten Teil der sekundären Rad. mesent, auch an der hinteren Bauchwand. Aber der Fall ist ja schon in anderer Beziehung kontrovers, so daß ich ihn nicht heranziehen will.

Gewiß sind das Lücken im Bereich der Linie der sekundären Radix mes., ganz anders gelagert, als die öfter beschriebenen Löcher, die unmittelbar am Darm, oder wenigstens diesem näher liegen als dem Ansatz an die hintere Bauchwand.

Wenn man überhaupt eine Erklärung für so gelagerte Defekte versuchen will, scheint mir diese am nächsten liegend.

Eine andere Gruppe, für die ein sehr interessanter Versuch, sie auf kongenitale Ursachen zurückzuführen, schon seit geraumer Zeit vorliegt, sind die eben erwähnten Defekte nahe am Darm, und zwar die dicht am Übergange des Ileum in Coecum gelegenen. Mit ihnen hat sich Treves schon im Jahre 1885 ausführlich beschäftigt.

Treves gibt für einen Teil der Mesenteriallöcher den traumatischen Ursprung zu: die seien meist schlitzförmig oder unregelmäßig und ihrem Sitz nach unbestimmt. Für die Mehrzahl könne diese Ätiologie nicht gelten. Denn sie sind meistens rund, sitzen im Mesenterium des unteren Ileumendes, um einen Teil des Randes geht nicht selten ein Ast der Art. mesent. sup.: das hatte ihm die Betrachtung aller ihm erreichbaren Museumspräparate gezeigt. Systematische Untersuchung des unteren Mesenterialendes bei einer großen Reihe von Föten ergab: oft umschreibt der Ramus ileocolicus der Art. mesent. sup. bei seiner Anastomose mit dem letzten Dünndarmast ein Mesenterialfeld von runder oder ovaler Gestalt; dieses Feld enthält kein Fett, keine Blutgefäße irgendwelcher Art, auch bei gut injizierten Präparaten, und keine Mesenterialdrüsen. Es ist eine gewöhnliche, aber nicht allgemeine Erscheinung bei reifen Früchten resp. Neugeborenen, doch bewahrt es auch im späteren Leben manchmal seinen Charakter. Von der Geburt bis zur Pubertät findet man das'Feld gewöhnlich von Schillinggröße, die Ränder durch die genannten Gefäße bezeichnet, manchmal etwas opaker.

Dies Feld hat nun wohl, meint Treves, offenbar Lage, Form und Größe des „mysteriösen Mesenteriallochs", und bei Atrophie des Bauchfells kann hier ein Loch leicht entstehen. 
Die beiden eigenen Beobachtungen, die Treves dabei anführt, sind schon in der Übersicht erwähnt (ganz am Schluß).

Tatsache ist nun, daß von den genau beschriebenen Fällen eine relativ große Zahl die Lücke da zeigen, wo sie nach Treves sitzen soll. Es sei nur darauf hingewiesen, daß allein Mitchell, der sich Treves anschließt, vier Fälle der Art gesammelt hat. Auch Lutier beruft sich direkt auf Treves.

Ich habe bisher Gelegenheit gehabt, bei Früchten aus den letzten Schwangerschafts- und Kindern aus den ersten Lebensmonaten in zwanzig Fällen nach dem "Trevesschen Feld" zu suchen. ${ }^{1)}$

14 mal war nichts davon zu sehen. 6 mal war der Gefäßbogen deutlich, einmal erschien auch das Mesenterium in seinem Bereich dünner, nie aber habe ich innerhalb des der Form nach deutlichen „Feldes" Fett, Gefäße und Lymphknoten vermißt. Auf Grund dieser kleinen Zahl kann ich aber kein Urteil fällen.

Treves spricht von einer "Atrophie des Bauchfells" als möglicher Vermittlerin für die Entstehung der Lücke im Felde, ohne sich über den Zusammenhang näher auszulassen.

Man könnte sich einen solchen Vorgang theoretisch wohl konstruieren.

Wie oben erwähnt, tritt das Colon bei der Überkreuzung des Dünndarms an der Kreuzungsstelle früh in nahe örtliche Beziehungen zu ihm, die sich in der Folgezeit, wenn überhaupt, jedenfalls nur in sehr bescheidenen Grenzen ändern. Zu der Zeit ist aber der nach rechts herübergetretene Teil des Dickdarms noch unverhältnismäßig klein: das Cöcum liegt noch dicht der Unterfläche der Leber an. Beide, der Darm und sein Gekröse, müssen noch erheblich wachsen, ehe sie das gewöhnlich von ihnen eingenommene Gebiet ausfüllen. Den weitesten Weg hat dabei das Cöcum zurückzulegen, und das Höhenwachstum des Gekröses ist am größten in der Rjchtung von der primären Radix mesenterii gegen den Ileumcolonwinkel. In dieser Richtung wird, wenn man so sagen darf, seine Wachstumsenergie am stärksten in Anspruch genommen. In dieser Linie liegt das Trevessche Feld. Nehmen wir nun den Fall an, daßs der Darm verhältnismäßig schneller wächst, als das zugehörige Gekrösestück - und mit solchen Un-

1) Für die gütige Überlassung des Materials bin ich dem Direktor des hiesigen anatomischen Instituts, Herrn Geheimrat L. Stieda, zu großem Dank verpflichtet. 
gleichmäßigkeiten im Wachstum rechnen wir doch, trotz aller Zweckmäßigkeit, die wir so gern überall sehen, in anderen Fällen auch -, dann wäre der Grund für die von Treves supponierte „Atrophie des Bauchfells" gerade an dieser Stelle gefunden.

Beidem - dem Versuch zur Erklärung der Lücken im Bereich der sekundären Radix mesenterii, wie diesem Versuch, Treves' Theorie zu stïtzen - lege ich keinerlei beweisende Bedeutung bei. Leider kommen wir gegenüber solchen Fragen aber doch für gewöhnlich nicht über das Theoretisieren hinaus.

Nur angedeutet sei im Anschluß hieran, daß ähnliche Differenzen im Wachstum der eng zueinander gehörigen Teile ähnliche Folgen haben könnten bei etwaigen Verschiebungen sekundärer Art. Gerade im Ileumcolonwinkel muß man, scheint mir, auch mit solchen rechnen. Cöcum und Proc. vermiformis entstehen als einfache Ausstülpungen an dem vom Mesenterium abgewandten Rande der Nabelschleife. Wenn also später Mesocöcum und Mesenteriolum vorhanden sind, läßt sich das wohl nur durch sekundäre Änderungen in den Lagebeziehungen der einzelnen Teile erklären. Die Vermutung liegt nahe, daß hier die ernährenden Gefäße gleichsam die Rolle von Leitbändern spielen, daß es sich sozusagen um Gefäßfalten handelt: das Material aber für diese Falten gibt das Mesenterium her, denn in ihm verlaufen die Gefäße. -

In gewissem Sinne auch für den kongenitalen Ursprung der Mehrzahl der Mesenteriallöcher läßt sich, wie schon angedeutet, schließlich der Umstand verwerten, daß andere, widerspruchslos als kongenital anerkannte Anomalien geradezu auffallend häufig daneben gefunden werden.

Nicht weniger als elfmal begegnen wir solchen Komplikationen: dreimal fanden sich Meckelsche Divertikel, die in den beiden genauer berichteten Fällen (Atherton und Pye Smith; Goltdammer macht leider keine Angaben) in direkter örtlicher Beziehung zu dem Mesenterialloch standen; zweimal (Remboldt und Thursfield) wird als Todesursache Volvulus duodeni angegeben, was eine andere Mesenterialanomalie voraussetzt; dazu kommt der Fall von Trendelenburg mit Mesent. ileo-coeci commune - wodurch da freilich das ganz merkwürdig geformte und lokalisierte Loch nicht erklärt wird; ebenfalls zweimal (Perth und Parsons) finden wir zwei Löcher, in dem Fall von Parsons sogar in einem „überzähligen“ Mesosigmoideum; schließlich gehören hierher die Fälle von Billroth und Hinterstoisser mit doch 
wahrscheinlich angeborenen Leistenbrüchen (beide rechts!), und denen darf man als elften wohl anschließen den Fall von Blandin mit Hernia foram. Winslowi: mag die auch erst intra vitam gewachsen, vielleicht sogar erst entstanden sein, ist doch eine primäre Anomalie in der Konfiguration des betr. Gebiets zu vermuten.

Das klinische Bild, das Einklemmungen in Mesenteriallöchern bieten können, ist so vielgestaltig, daß jeder Versuch, besondere Merkmale, die diagnostisch verwertet werden könnten, herauszufinden, vergeblich bleiben muß. Art und Intensität der Erscheinungen und damit Art und Daller des Verlaufs, ebenso die anatomischen Bilder des eingeklemmten Darms schwanken geradezu zwischen den Extremen dessen, was wir bei Darmverschlüssen überhaupt zu sehen bekommen. Fällen, die sich über 15 (Rokitansky), 16 (Parsons), 18 Tage (Lévêque) erstreckten, vor allem dem Fall von Trendelenburg, wo noch am 18. Tage der Darm nicht gangränös gefunden wurde, stehen andere gegenüber, die in 48 (Adelmann), 33 (Lancereaux), 27 (Bauer), ja 18 Stunden (Perth) tödlich verliefen. Der am schnellsten verlaufene Fall (Atherton) ist in dieser Hinsicht nicht zu den Verschlüssen durch Mesenteriallöcher, sondern zu den Divertikelverschlüssen zn rechnen: hier fand sich bei der Operation sechs Stunden nach Beginn der Erscheinungen das Meckelsche Divertikel gangränos. Das Loch im Mesenterium wurde gar nicht gefunden, so daß es angesichts der Drehung, die das Divertikel zeigte, doch fraglich bleiben muß, ob die Öffnung wirklich beteiligt gewesen war. Dafür spricht freilich die Wahrnehmung, daß der Darm mit dem Divertikel sich etwas schwer vorziehen ließ. (Vgl. die Anmerkung zur Krankengeschichte und die beiden Schemata dort.)

Der Beginn der Erkrankung schon zeigt große Verschiedenheiten. Interessant sind in dieser Beziehung die Vorläufer - oft sind es geradezu typische Einklemmungen, die aber spontan oder wenigstens ohne Operation wieder zurückgehen, in anderen Fällen ist nur von Neigung zur Obstipation die Rede; mehrfach zeigt sich eine Zunahme dieser Erscheinungen, bis es endlich zur völligen Einklemmung kommt. Weiter kommen, teils mit solchen Vorläufern, teils ohne sie, Fälle vor, die eine Gelegenheitsursache erkennen oder wenigstens vermuten lassen: reichliche Mahlzeit, sehr kaltes Bad, sehr kaltes Getränk, anstrengende Arbeit - was ja 
Die angebor. u. (nicht operat.) erworbenen Lücken u. Spalten d. Mesenter. 427

bei jeder Art von Darmverschluß auch vorkommt. Ihnen stehen wieder andere gegenüber, in denen die bis dahin vollständig gesunden Menschen ohne jeden ersichtlichen Grund ganz plötzlich, ja sogar im ruhigen Schlaf, von den Erscheinungen überfallen werden.

Einen gewissen Fingerzeig freilich geben diese verschiedenen Arten des Beginns dafür, wie wir uns den Eintritt der Schlinge in die Öffnung zu denken haben. Bei körperlicher Anstrengung wird der Darm in die Öffnung hineingepreßt, wie z. B. wohl in dem Fall von Brambilla (der freilich auch die Entstehung des "Risses" selbst so erklären wollte); in anderen Fällen wieder z. B. in dem von Lancereaux, dessen Kranke schon drei Wochen vorher in Beobachtung gewesen war - kann man füglich nur Änderungen in der Lagerung des Darms in Verbindung mit der Wirkung der Peristaltik anschuldigen. Vielleicht wirkt, in den Fällen von Erkrankung im Schlafe, längeres Einhalten derselben Körperlage mit. Alle Möglichkeiten können hier nicht erörtert werden.

Besser als das kann die übergroße Mannigfaltigkeit des Verlaufs durch alle seine Phasen verständlich machen ein Blick auf die verschiedenen anatomischən Formen der Einklemmung, denen wir hier begegnen. Blecher hat sie unter vier Typen einzuordnen gesucht:

1. Die dem Loch benachbarte Schlinge kann eintreten, bis ihr einer Fußpunkt dem Übergang des zum Loch gehörigen Darms in den durchgetretenen entspricht. Ist die Schlinge an einem Ende fixiert, wie z. B. das Ileum am Cöcum, so kann hier eine Abknickung oder Drehung um die Darmachse eintreten. Es kommt nicht immer gleich zum Verschluß, der sogar erst nach sehr langer Zeit (im Falle von Rokitansky erst drei Jahre danach [?]) eintreten kann.

Dieser "einfache" Verschluß kann auch neben 2. und 3. vorkommen.

2. Einklemmung wie beim Bruch, Koteinklemmung oder auch elastische Einklemmung.

3. Durch eine größere Öftnung tritt eine Schlinge und dreht sich dann um ihre Mesenterialachse: erst beides zusammen macht den Rücktritt der Schlinge unmöglich und führt zum Verschluß.

4. Nach seinem eigenen Fall stellt Blecher zu diesen schon bekannten Formen noch die der Drehung der zum Loch gehörigen 
Schlinge selbst. So entstehe ein Volvulus „mit doppeltem Stiele, die beide in der Mesenterialebene liegen".

Wilms unterscheidet

1. Elastische Einklemmung.

2. Durchtritt von Darm mit Knickung oder Drehung: Obturation.

3. Durchtritt in Verbindung mit Volvulus: das sei dann kein Umschlagen des Darms, sondern hänge lediglich vom Zufall ab, wie gerade die Schlinge beim Beginn des Durchtritts gelegen habe.

4. Der gleiche Typus von Blecher.

Die Unterschiede beschränken sich im wesentlichen auf die Gruppierung. Im ganzen schließe ich mich Blecher an, möchte nur Wilms darin beitreten, daß in der Verbindung mit Volvulus der durchgetretenen Schlinge (es ist viermalige Drehung beobachtet) nur ein zufälliges Zusammentreffen zu sehen ist. Besonders betonen muß ich aber, daß Blecher in seinem ersten und vierten Typus die Formen beschreibt, die für die Einklemmung in Mesenteriallöchern geradezu charakteristisch sind. Namentlich die von Blecher an seinem Fall zum erstenmal erkannte Form kommt wohl nur hier vor: die doppelte Drehung eines Dünndarmabschnittes um die Darmachse. Die kann nur die Schlinge treffen; deren Mesenterium das Loch enthält, wenn sie sich selbst durch dieses hindurchdreht. Ob dazu nun noch, wie in Blechers Fall, ein Volvulus dieser Schlinge im eigentlichen Sinne, d. h. Drehung um die Mesenterialachse, hinzutritt oder nicht, ist nebensächlich.

$\mathrm{Ob}$ es bei solcher Einklemmung der Schlinge in ihrem eigenen Mesenterium zur Strangulation kommt oder nicht, hängt wesentlich von der Größe der Öffnung ab - die in gleicher Weise maßgebend dafür ist, ob elastische Einklemmung der eigenen, der benachbarten oder einer entfernteren Schlinge zustande kommt.

Der ja auch in anderer Hinsicht eigenartige Fall von Trendelenburg z. B. zeigte bei seit 18 Tagen bestehender doppelter Durchdrehung der dem einen (aboralen) Rand des großen Schlitzes entsprechenden großen Darmpartie (unterstes Fünftel des Dünndarms, Cöcum und Colon ascendens!) noch ganz intakten Darm. Einfache Rückdrehung ließ sofort den Kotlauf wieder in Gang kommen. Der endliche fatale Ausgang fiel wohl einer Dehnungsperforation des Cöcums zur Last - ein Zeichen, daß der Darm über der Durchdrehung vielmehr geschädigt war wie der durchgedrehte 
Die angebor. u. (nicht operat.) erworbenen Lücken u. Spalten d. Mesenter. 429

selbst, was ja auch die viel stärkere Erweiterung des ersteren anzeigte.

Ein gutes Beispiel für die Durchdrehung der zugehörigen Schlinge ist weiter der Fall von Kellett Smith: die Schlinge war um ihre beiden Fußspunkte - der proximale und distale Übergang vom gekröselosen zum normalen Darm haben die gleiche Bedeutung wie die Fußpunkte der Flexura sigmoidea - herumgedreht; daß hier nach vier Tagen noch keine Gangrän bestand, hängt wohl damit zusammen, daß das Loch im Verhältnis zur Schlinge relativ groß war.

In dem Fall von Lutier bildete die Schlinge infolge Näherung der Fußpunkte eine lange Schleife - so geradezu eine Flex. sigmoidea nachahmend. Hier bestand neben der Durchdrehung noch eine Drehung um die Mesenterialachse.

Wird die zugehörige Schlinge an beiden Enden um die Darmachse gedreht, so geschieht das bei der benachbarten nur an dem Ende, das an das Loch grenzt: bei der nächsthöheren am unteren. bei der nächsttieferen am oberen Ende.

Da häufig das Loch dicht am unteren Ileumende sitzt, wird dieser Darmteil besonders häufig eingeklemmt. Dabei macht sich die Fixation gegen das Cöcum, auf die auch Blecher besonders hinweist, geltend. Jas unterste Ileumstück ist gedehnt, platigedrückt. So beschreibt Greig Smith sehr anschanlich, daß er es zunächst, wegen seiner Dünne und Blässe, für den Wurmfortsatz hielt, der aber bald dicht rechts davon in normalem Zustande gefunden wurde.

Dieser Vorgang, daß das unterste Ileumende geradezu hineingezogen wird, bildet wohl eine vorzügliche Illustration für den Hergang solcher Einklemmungen, wie ihn Wilms geschildert hat. Es ist die durch die Einengung angeregte Peristaltik, die den Darm geradezu hineinzieht in das Hindernis, soweit er zu folgen vermag. In derselben Weise ist die Tatsache zu erklären, daß manchmal erstaunlich große Darmabschnitte durch verhältnismäßig kleine Löcher treten.

Man kann den Vorgang so umschreiben: das von außen den Darm umgebende Hindernis (Ring) verhält sich gegenüber den Darmbewegungen ähnlich wie ein der Innenwand des Darms fest anliegender Körper, der Darm sucht es nach unten abzustreifen. Das Hindernis ist hier aber der unbewegliche, der Darm der bewegliche Teil, also verändert er seinen Ort, kriecht scheinbar in das Hindernis hincin, oder wird hineingezogen - wie man es aus- 
drücken will; eigentlich aber streift er das Hindernis an sich herunter.

Die einzelnen Fälle zeigen noch eine Fülle interessanter Details, auf die nicht näher eingegangen werden kann, auch für die Auffassung vom Mechanismus der verschiedenen Formen der Einklemmung. Ich kann hierfür nur anf die Auszüge verweisen. -

Daß es um die Diagnose nicht sonderlich bestellt ist, kann bei der Vielgestaltigkeit der Erscheinungen nicht wundernehmen. In keinem Fall ist die Art des Verschlusses erkannt worden. Interessant sind die begreiflichen Verwechselungen mit Perityphlitis (Atherton, Anderson). So detaillierte Diagnosen, wenigstens bezüglich des Sitzes des Hindernisses, wie die von Ridge und Hilton oder von Hirsch, sind nicht gewöhnlich; freilich lagen die Fälle auch günstig dafür.

Aus den Ergebnissen der operativen Behandlung etwa auf ihre allgemeinen Aussichten oder auf den Grad der Gefahr dieser Einklemmungen schließen zu wollen, wäre verfehlt. Der eine oder andere von den operierten Fällen mag auch verschleppt worden sein, hätte vielleicht gerettet werden können. Damit steht es hier aber wohl nicht schlechter und nicht besser als bei allen anderen Formen von Darmverschluß. Erwägt man die Bedingungen, unter denen die einzelnen Fälle zur Operation kamen, sind die Resultate nicht direkt schlecht zu nennen, obgleich von den 18 operierten Fällen nur fünf geheilt sind (Franklin, Greig Smith, Kellett Smith, Blecher, Pye Smith). Sie sind am dritten bis fünften Tag operiert, also verhältnismäßig spät. - Ein Teil der anderen ging, wie so oft, zu spät zl, in anderen bestanden Komplikationen, die den Versuch der Rettung schlechterdings vereiteln mußten. - Daß bei einer wirklich festen Einklemmung nur die Operation helfen kann, braucht gar nicht erst gesagt zu werden. Auf der anderen Seite scheinen die Aussichten für spontanen Rückgang nicht ganz schlecht, wenn man erwägt, daß in nicht wenigen Fällen eine z. T. beträchtliche Zahl von Attacken vorausgegangen war, die man als Einklemmungen in dem Mesenterialloch auffassen muß. Das darf natürlich nicht zum Zuwarten verleiten in Fällen, die bedrohliche Erscheinungen zeigen.

Alles in allem: alle Möglichkeiten, alle Schwierigkeiten, alle Zweifel, alle Enttäuschungen, denen wir in dem großen Gesamtgebiet des Darmverschlusses begegnen, sehen wir hier im Rahmen einer seltenen, fast als Merkwürdigkeit angesehenen Form wiedererstehen. Gewiß wird sich anch hier noch manche Frage der 
Die angebor. u. (nicht operat.) erworbenen Lücken u. Spalten d. Mesenter. 431

Lösung näher bringen lassen. Diese Zusammenstellung wollte nur dazu beitragen, das Material dazu zu sammeln und etwas. zu sichten.

\section{Literaturverzeichnis.}

1. Adelmann, Beiträge zur chirurgischen Pathologie und Therapie der Ernährungsorgane. I. Die Verschließung der Därme innerhalb der Bauchhöhle. (Prager) Vierteljahrschr. f. prakt. Heilkde. 1863, II, 32.

2. Anderson, A., Case of internal strangulation through a hole in the mesentery. Brit. med. Journ. 1903, I, 489.

3. Arbuthnot Lane, W., Case of strangulation of the bowel through a hole in the mesentery. Brit. med. Journ. 1890, I, 890.

4. Sir Astley Cooper, Anatomische Beschreibung und chirurgische Behandlung der Unterleibsbrüche. Nach der II., von C. Aston Key besorgten Ausgabe. Aus dem Englischen übersetzt. (Von R. Froriep.) Weimar 1833 . S. 210.

5. A therthon, A. B., Case of Strangulation of a loop of ileum through a hole in the mesentery, with a Meckel's diverticulum attached. Brit. med. Journ. 1897, II, 975.

6. Bauer, Internal strangulation of small intestine through an opening in the mesentery; death within 27 hours. (New York) Medical Record II, 27. 1867. Virchow-Hirsch 1867, II, 462.

i. Billroth, Chirurgische Erfahrungen. Zürich 1860-1867. Archiv f. klin. Chir., Bd. 10, S. 511. Eine innere Einklemmung. Laparotomie.

8 . Blecher, Über Ileus, bedingt durch seltenere Formen von Volvulus. Deutsche Zeitschr. f. Chir., Bd. 61, S. 48.

!) Dittrich, Beiträge zur pathologischen Anatomie. (Prager) Vierteljahrsschr. f. prakt. Heilkde. 1847, I, 120 ff. (Fall 2).

10. Franklin, George Cooper, Internal strangulation in an aperture of the mesentery; acute intestinal obstruction; operation; recovery. Lancet 1894, I, 334.

11. Goltd ammer, E., Über Ileus. Berl. klin. Wochenschr. 1899, Nr. 10, S. 198.

12. Greig Smith, A case of intestinal obstruction through a hole in the mesentery, associated with volvulus and haemorrhage into the abdominal cavity; operation; recovery. Brit. med. Journ. 1897, II, 1022.

13. de Haën, Ratio medendi. Pars XI. De ileo morbo. S. 141. Zit. nach Treitz, l. c. S. 10 (l).

14. Hinterstoisser, Herm., Jahresbericht der chirurgischen Abteilung des allgem. Krankenhauses in Teschen. Teschen 1902.

15. Hirsch, Ein Fall von inverer lncarceration des Darms, bedingt durch eine anomale Öfnung im Mesenterium. St. Petersb. med. Wochenschr. 1880, Nr. 32, S. 263.

16. Jobert, Traité théorique et pratique des maladies du Canal intestinal. Paris 1829. Bd. I, S. 522. (Beobachtung von Blandin,; 
17. Kaurin, Ileus. Tidskr. for praktisk Med. 1883, S. 1. Virchow-Hirsch 1883, II, 203 (Fall 2).

18. Kellett-Smith, J. Stanley, A case of strangulation of the ileum by passage through a congenital mesenteric hole; operation; recovery. Iancet 1897, II, 1111.

19. Kü m mell, H., Über Laparotomie bei innerer Darmeinklemmung. Deutsche med. Wochenschr. 18\$6, S. 191.

20. Lancereaux, E., Contribution à l'ctude de quelques affections du péritoine. Arch. gén. de Méd. VII ème Sér., T. II. (141 Vol. de la Coll.) 187s, II, 569 .

21. Lévêque, Emile, Etranglement de l'intestin grêle à travers une déchirure du mésentère, à son insertion antérieure, compliqué de torsion (volvulus) et de rétrécissement. Progrès méd. 188t, Bd. XII, S. 165.

22. Lilienthal, Howard, Intraperitoneal hernia with strangulation. Annals of Surgery 1897, Sept.

23. Lutier, A., Occlusion intestinale par hernie iuterne et volvulus de l'iléon dans l'aire mésentérique de Treves. Bull. et Mém. de la Soc. anat. de Paris. LXXVe Année. Gème Série, T. II, S. 639, 1900.

24. Mauclaire, Occlusion intestinale par étranglement de l'intestin dans une grande perforation du mésentère. Bull. et Mém. de la Soc. anat. de Paris. LXXIVe Année. 6ème Série, T. I, S. 247, 1899.

25. Mitchell, I, ouis J., Strangulated internal hernia through a mesenteric hole. Annals of Surgery, Bd. 30, S. $505,1899$.

26. Nélaton, Fälle von Laparo-Enterotomie. Aus der Klinik Nélatons mitgeteilt von Veillard. L'Union méd. 185̄7, Nr. 89, 91 u. 93. Ref. Schmidts Jahrb., Bd. 96, S. 214.

27. v. Ofenheim, Ernst, Über einen Fall von Volvulus infolge eines Mesenterialdefekts. Diss. Leipzig 1902. Mit 2 Tafeln. (Fall von Trendelenburg.)

28. Parsons, W. B., A case of hernia through a congenital opening in as abnormal fold of peritoneum. Lancet 1899, II, 1439.

29. Pye-Smith, R. J., A case of acute intestinal obstruction by a fish-fin lodging above a Meckel's diverticulum; laparotomy; recovery. Lancet 1899, I, 47\%.

30. Ridge, Jos., Case of strangulation of the jejunum released by gastrotomy; with observations on the diagnosis and treatment of intestinal obstructions within the abdomen. Hunterian Society 1S. Jan. 1854. Zit. nach Treitz, S. $91 \mathrm{ff}$.

31. Spörer, Beobachtungen über den Ileus. Verm. Abhandl. aus dem Gebiete d. Heilkde. von einer Gesellsch. prakt. Ärzte zu Petersburg. 8. Sammlung. Ref. Berl. med. Centralztg. 185̄ō, Nr. 4l, S. 323.

32. Strömborg, Et fall af hastigt förlöpande volvulus. Finska läkaresällsk. handlingar. Bd. 29, S. 395. Virchow-Hirseh 1S88, II, 276.

33. Thursfield, H., An unusual case of intestinal obstruction. Brit. merl. Journ. 1901, I, 1269.

34. Toldt, C., Bau und Wachstumsveränderungen der Gekröse des menschlichen Darmkanales. Denkschriften der Kaiserl. Akademie d. Wissensch,, Bd. 41, II. T., S. 1. Wien 1879. 
Die angebor, u. (nicht operat.) erworbenen Lücken u. Spalten d. Mesenter. 433

35. Treitz, W., Hernia retroperitonealis. Ein Beitrag z. Geschichte innerer Hernien. Prag 1857.

36. Treves, Frederick, Lectures on the anatomy of the intestinal canal and peritoneum in man. Brit. med. Journ. 1885, II, 415, 470.

37. Vitrac et Laubie, Contusion de l'abdomen. Journ. de Méd. de Bordeaux 1897, No. 15. Ref. Hildebrands Jahresber.

38. Wilms, M., Der lleus. Deutsche Chirurgie, Liefg. $46 \mathrm{~g}$. Stuttgart 1906. S. $352 \mathrm{ff}$.

Weitere Literatur bei Blecher.

39. Albers, Caspar's Wochenschr. 1837, S. 201.

40. Brambilla, Commentarii de Bononiensi scientiarum etc. Bologna 1731.

41. Davies, Boston med. and surg. Journ. 1814. Dec.

42. Hieuermann, Abhandlungen der wichtigsten chirurgischen Operationen, Bd. 1, S. 627, Kopenhagen 1778.

43. Loebl, Zeitschr. d. k. k. Ges. d. Ärzte zu Wien 1844, I, 154.

44. Morestin, Bull, et Mém. de la Soc. anat. de Paris 1900, No. 5 .

45. Remb oldt, Oesterr. Zeitschr. f. prakt. Ärzte. 1865, I, 109.

46. Rokitansky, Oesterr. Jahrbücher der Med. X, 669. 1836.

Von folgenden Fällen habe ich nur die Nachweise ermitteln können, aber weiter nichts Genaueres:

47. Barling, Med. Press. 21. Sept. 1898.

48. Besnier, De l'étranglement interne de l'intestin. Paris 1860 (S. erste Textanmerkung.)

49. Flenry, Arch. gén. de Méd. II. Sér., T. XV, S. 102. (Nach Leichtenstern, v. Ziemssens Handb., Bd. VII, 2, S. 415, ebenso wie der folgende Fall zweifelhaft bezüglich der Zugehörigkeit.)

50. Habershon, On diseases of the abdomen. London 1862.

51. Hofmokl, Klin. Zeit- und Streitfragen, Bd. 6, H. 4 u. 5 .

52. Hutchins on, Lancet 1898, I, 641.

53. Kakeles, (New York) Med. Record. 1888, Nr. 24.

54. Lockwood, Transact. of the clinical Soc. of London. Bd. 13. 1897.

55. Monro, Observations on crural hernia. Tafel I, Fig. 1. (Zit. nach Treitz, S. 91.) 